Central European Science Journals
Central European Journal of Mathematics

Central European Science Journals

DOI: $10.1007 / \mathrm{s} 11533-005-0002-5$

Research article

CEJM 4(1) 2006 5-33

\title{
Category with a natural cone
}

\author{
Francisco J. Díaz*, Sergio Rodríguez-Machín \\ Departamento de Matemática Fundamental, \\ Universidad de La Laguna \\ C/ Astrofísico Francisco Sanchez s/n, 38271, La Laguna, España
}

Received 2 March 2005; accepted 21 September 2005

\begin{abstract}
Generally, in homotopy theory a cylinder object (or, its dual, a path object) is used to define homotopy between morphisms, and a cone object is used to build exact sequences of homotopy groups. Here, an axiomatic theory based on a cone functor is given. Suspension objects are associated to based objects and cofibrations, obtaining homotopy groups referred to an object and relative to a cofibration, respectively. Exact sequences of these groups are built. Algebraic and particular examples are given. We point out that the main results of this paper were already stated in [3], and the purpose of this article is to give full details of the foregoing. (c) Central European Science Journals Warsaw and Springer-Verlag Berlin Heidelberg. All rights reserved.
\end{abstract}

Keywords: Category, algebraic homotopy theory, cone construction MSC (2000): 55U35, 18C, 55P05, 55P40, 55Q05

\section{Introduction}

In this paper, basic properties of the topological cone are generalized to create a homotopy theory on arbitrary categories. The cone of a topological space is obtained by collapsing the base of its cylinder to a single point. The real numerical product defined on the unit interval originates a projection of the double cone onto the simple cone. The natural inclusions in the double cone, together with this projection, make the double cone behave like a cylinder of the simple cone.

Dual standard constructions in the sense of P.J. Huber [9] generalize properties of the topological cone. In the present paper, some axioms about cofibrations given by H.J. Baues [1] in categories with a natural cylinder are adapted to the point of view described

* E-mail: fradiaz@ull.es 
above: collapsing the base of the cylinder to a single point. The axiomatic theory obtained in this way allows one to obtain homotopy groups through suspensions of based objects and cofibrations. Also, exact homotopy sequences of these groups can be created.

The main axiomatic homotopy theories based on a cone are particular cases of this theory: Huber [9] obtained homotopy groups and exact sequences of them on projective and on injective homotopy theories and pointed topological spaces. However, in general, the associated semisimplicial complexes do not verify the Kan extension property. So, the existence of homotopy groups cannot be guaranteed. Kleisli [12] defined homotopy theory on additive categories with additional properties. Seebach [18] created injective homotopy theory. Finally, Rodríguez-Machín [16] worked on additive categories.

Objects in categories with a natural cylinder in the sense of Baues [1] are cofibrants, and the model categories defined by D.G. Quillen [17] give an autodual theory. In general, these facts are not true in categories with a natural cone. Hence, there are categories with a natural cone that are not derived from categories with a natural cylinder (it suffices that the category does not have an initial object). On the other hand, it is possible to develop a dual theory (category with natural cocone). If the category is additive with finite limits and colimits, cofibrations and fibrations are suitably defined, and there is compatibility between the cone and cocone structures, then a proper closed model category structure is induced (see [5]). However, this result is not available for general categories, since weak equivalences cannot be defined.

The well-known homotopy theories are examples of this axiomatic theory or of its dual: the classical homotopy of topological spaces, pointed topological spaces and chain complexes [10]; projective and injective homotopy theories of R-modules [8]. Others less known are examples too: some tensorial homotopy theories and the proper homotopy theory of exterior spaces [6].

Dedicated to Sergio, wherever he is.

\section{Notation}

The following categorical notation will be used in this paper.

Given functors $\mathbf{B} \stackrel{E}{\rightarrow} \mathbf{C} \stackrel{F, G}{\rightarrow} \mathbf{D} \stackrel{H}{\rightarrow} \mathbf{E}$ and a transformation $t: F \rightarrow G$, then the transformations $t * E: F E \rightarrow G E$ and $H * t: H F \rightarrow H G$ will be denoted by $t_{E}$ and $H t$, respectively. When there is no possibility of confusion, the morphism $t_{X}: F X \rightarrow G X$ will be simply denoted by $t$, for every object $X$.

The pushout object of two morphisms $f$ and $g$ will be denoted by $P\{f, g\}$. The induced morphisms will be denoted by $\bar{f}:$ codom $g \rightarrow P\{f, g\}$ and $\bar{g}:$ codom $f \rightarrow$ $P\{f, g\}$. Given a morphism $f$, if the notation $\bar{f}$ has been used, $\widetilde{f}$ will denote the other morphism induced by $f$ in a pushout. In particular, if $f=g$, then $\bar{f}$ and $\tilde{f}$ will denote the morphisms $\bar{f}$ and $\bar{g}$, respectively.

Given morphisms $r$ and $s$ verifying $r f=s g$, the unique morphism $h$ such that $h \bar{g}=r$ and $h \bar{f}=s$ will be denoted by $\{r, s\}$. If codom $f$ (resp. codom $g$ ) is a pushout object, the component $r$ (resp. $s$ ) has an expression of the form $\left\{r_{1}, r_{2}\right\}$ (resp. $\left\{s_{1}, s_{2}\right\}$ ). In this 
case, the morphism $\{r, s\}=\left\{\left\{r_{1}, r_{2}\right\}, s\right\}$ (resp. $\left\{r,\left\{s_{1}, s_{2}\right\}\right\}$ ) will be frequently denoted by $\left\{r_{1}, r_{2}, s\right\}$ (resp. $\left\{r, s_{1}, s_{2}\right\}$ ). In this way, expressions of the type $\left\{h_{0}, h_{1}, \ldots, h_{n}\right\}$ can appear.

Given two pushout objects $P\{f, g\}$ and $P\left\{f^{\prime}, g^{\prime}\right\}$, and three morphisms $r:$ codom $f \rightarrow$ codom $f^{\prime}, s:$ codom $g \rightarrow$ codom $g^{\prime}$ and $t: \operatorname{dom} f=\operatorname{dom} g \rightarrow \operatorname{dom} f^{\prime}=\operatorname{dom} g^{\prime}$ verifying $r f=f^{\prime} t$ and $s g=g^{\prime} t$, we will denote the unique morphism $\left\{\overline{g^{\prime}} r, \overline{f^{\prime}} s\right\}$ by $r \cup s$. If there is no possibility of confusion, expressions of the type $h_{0} \cup h_{1} \cup \ldots \cup h_{n}$ will be used.

The pullback object of two morphisms $f$ and $g$ will be denoted by $P<f, g>$, with induced morphisms $\bar{f}$ and $\bar{g}$, respectively. The morphisms induced by the universal property will be denoted by $<r, s>$ or $r \cap s$.

Finally, the set of extensions of a morphism $u: B \rightarrow X$ relative to another morphism $i: B \rightarrow A$ is defined by $\operatorname{Hom}(A, X)^{u(i)}=\{f: A \rightarrow X / f i=u\}$

\section{Category with a natural cone}

Here, we state the minimal structure that a category must have in order to obtain a homotopy theory via a cone functor together with a class of distinguished morphisms, called cofibrations, that verify properties obtained by generalizing certain homotopy properties of topological cofibrations.

The concepts of nullhomotopic continuous function and contractible topological space are generalized. Also, the concepts of contractible cofibration and pointed $C$-category are introduced. Finally, relative homotopy theory is defined for cofibrations with codomain a cone object, and its basic properties are studied.

Definition 3.1. A category with a natural cone, or $C$-category, is a category $\mathbf{C}$ together with a class "cof" of morphisms in $\mathbf{C}$, called cofibrations and denoted by $\longmapsto$, a functor $C: \mathbf{C} \rightarrow \mathbf{C}$ which will be called the cone functor, and natural transformations $\kappa: 1 \rightarrow C$ and $\rho: C C \rightarrow C$, denominated inclusion and projection respectively, satisfying the following axioms:

C1. Cone axiom. $\rho \kappa_{C}=\rho C \kappa=1_{C}$ and $\rho \rho_{C}=\rho C \rho$.

C2. Pushout axiom. For any pair of morphisms $A \stackrel{i}{\leftarrow} B \stackrel{f}{\rightarrow} X$, where $i$ is a cofibration, there exists the pushout square

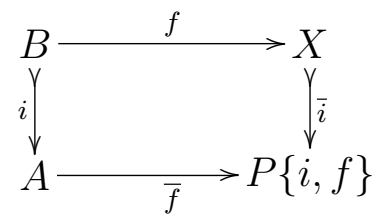

and $\bar{i}$ is also a cofibration. The cone functor carries this pushout diagram (called a cofibrated pushout) to a pushout diagram, that is $C(P\{i, f\})=P\{C i, C f\}$.

C3. Cofibration axiom. For each object $X$ the morphisms $1_{X}$ and $\kappa_{X}$ are cofibrations. The composition of two cofibrations is a cofibration. Moreover, there is a retraction 
for the cone of each cofibration (that is, if $i: B \longmapsto A$ is a cofibration, there is a morphism $r: C A \rightarrow C B$ such that $r(C i)=1)$. This last property is called the nullhomotopy extension property (NEP).

C4. Relative cone axiom. Given a cofibration $i: B \longmapsto A$, the morphism $i_{1}=\{C i, \kappa\}$ : $\Sigma^{i}=P\{\kappa, i\} \longmapsto C A$ is also a cofibration. The object $\Sigma^{i}$ is called the relative cone of $i$.

The following properties are consequences of Definition 3.1.

Every isomorphism $f: X \rightarrow Y$ is a cofibration, since $f=\overline{1_{X}}=\widetilde{1_{X}}: X \rightarrow$ $P\left\{1_{X}, 1_{X}\right\}=Y$. The cone of every cofibration $i$ is a cofibration, since $\bar{i}$ and $i_{1}=\{C i, \kappa\}$ are cofibrations and $C i=i_{1} \bar{i}$. Therefore, the cone functor carries cofibrated pushouts into cofibrated pushouts. Hence, the natural transformations $\kappa$ and $\rho$ induce unions of themselves, $\kappa \cup \kappa$ and $\rho \cup \rho$, between cofibrated pushouts. Moreover, given a morphism $\{f, g\}$ whose domain is a cofibrated pushout, then $C\{f, g\}=\{C f, C g\}$.

On the other hand, by the inductive use of the relative cone axiom, every cofibration $i: B \longmapsto A$ generates for each natural number $n$ a cofibration $i_{n}=\left(i_{n-1}\right)_{1}: \Sigma^{i_{n-1}} \rightarrow C^{n} A$, with $i_{0}=i$. Every commutative square $f i=i^{\prime} g$ relating cofibrations $i: B \longmapsto A$ and $i^{\prime}: B^{\prime} \longmapsto A^{\prime}$ induces, for each $n$, a morphism $\Sigma_{n}(f, g)=C^{n} g \cup C^{n-1} f \cup \ldots \stackrel{(n)}{ }\left(. \cup C^{n-1} f\right.$ : $\Sigma^{i_{n-1}} \rightarrow \Sigma^{i_{n-1}^{\prime}}$.
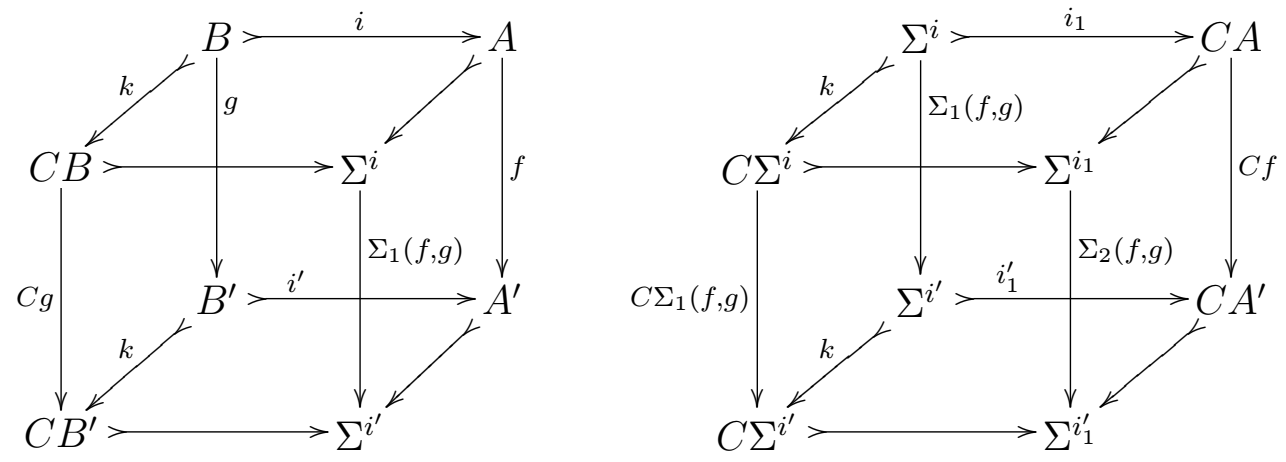

Remark 3.2. Given a cofibration $i$, the expression $\left\{f_{n}, f_{n-1}, \ldots, f_{0}\right\}$ symbolizes a morphism with domain the object $\Sigma^{i_{n}}$ if and only if the expression $\left\{f_{n}, f_{n-1}, \ldots, f_{1}\right\}$ symbolizes a morphism with domain $C \Sigma^{i_{n-1}}$, the domain of $f_{0}$ is $C^{n} A$ and $f_{0} i_{n}=\left\{f_{n}, f_{n-1}, \ldots, f_{1}\right\} \kappa_{\Sigma^{i_{n-1}}}$. By applying this fact to the expressions $\left\{f_{n}, \ldots, f_{m}\right\}$, for $0 \leq m \leq n$, and observing that the cone functor carries cofibrated pushouts into cofibrated pushouts, it is concluded that an expression of the type $\left\{f_{n+1}, f_{n}, \ldots, f_{0}\right\}$ symbolizes a morphism with domain $\Sigma^{i_{n}}$ if and only if $f_{r} C^{n} i=f_{n+1} C^{r} \kappa$ for $0 \leq r \leq n$ and $f_{r} C^{s} \kappa=f_{s+1} C^{r} \kappa$ for $0 \leq r \leq s \leq n-1$.

Remark 3.3. The following result gives an useful tool to know when a morphism is a cofibration between pushout objects. Given the commutative cubical diagram 


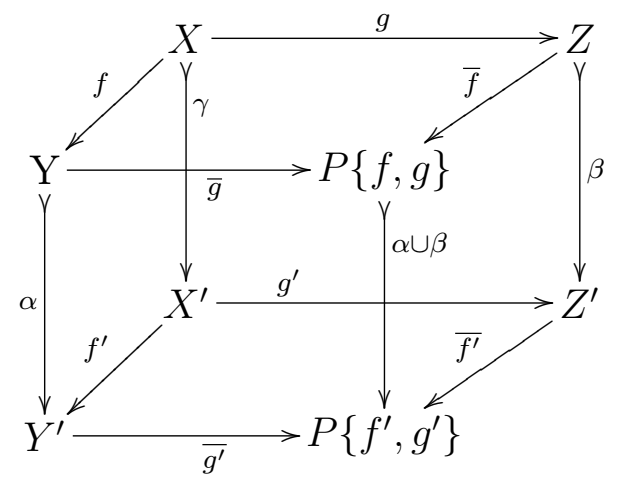

where the top and bottom faces are pushouts and $\alpha, \beta, \gamma$ are cofibrations. If $\left\{g^{\prime}, \beta\right\}$ : $\mathrm{P}\{\gamma, g\} \rightarrow \mathrm{Z}^{\prime}$ or $\left\{f^{\prime}, \alpha\right\}: \mathrm{P}\{\gamma, f\} \rightarrow \mathrm{Y}^{\prime}$ is a cofibration, then so is $\alpha \cup \beta$.

Definition 3.4. A morphism $f: X \rightarrow Y$ is said to be nullhomotopic (in symbols $f \simeq 0$ ) if there exists an extension $F$ of $f$ relative to $k$, that is, $F: C X \rightarrow Y$ such that $F k=f$. The morphism $F$ is called a nullhomotopy for $f$ (in symbols $F: f \simeq 0$ ). An object $\mathrm{X}$ is said to be contractible (in symbols $X \simeq 0$ ) when $1_{\mathrm{X}} \simeq 0$.

Observe that zero objects are contractible. Moreover, by $\mathbf{C 1}$ the cone of any object is contractible. By the naturality of $\kappa$, it is easily seen that a morphism is nullhomotopic if and only if it can be factored through a contractible object. Consequently, the composite of a morphism with a nullhomotopic morphism is nullhomotopic.

The above properties justify the use of "nullhomotopy" in the term NEP.

Theorem 3.5. Given a morphism $i: \mathrm{B} \rightarrow \mathrm{A}$, the following sentences are equivalent:

a) The morphism $i$ verifies the NEP.

b) Every nullhomotopic morphism $f: B \rightarrow X$ has a nullhomotopic extension rel. $i$.

c) Every nullhomotopic morphism $f: B \rightarrow X$ has an extension rel. $i$.

d) The inclusion $\kappa: B \rightarrow C B$ has an extension rel. $i$.

Proof. a) implies $b$ ) since by the NEP there exists a retraction $r$ for $C i$, and $\tilde{f}=F r \kappa$ is an extension of $f$ rel. $i$, where $F: f \simeq 0$.

Clearly $b$ ) implies $c$ ) and $c$ ) implies $d$ ), since the inclusion $\kappa$ is nullhomotopic.

d) implies $a$ ) since $r=\rho(C \tilde{\kappa})$ is a retraction for $C i$, where $\tilde{\kappa}$ extends $\kappa$ rel. $i$.

We point out that if $i: X \rightarrow Z$ verifies the NEP and $X \simeq 0$ or $Y \simeq 0$, then every morphism $f: X \rightarrow Y$ has an extension relative to $i$.

The notion "contractible" can be extended to cofibrations. Such contractible cofibrations will be the contractible objects in the category of pairs.

Definition 3.6. A cofibration $i: B \longmapsto A$ is said to be contractible when $B$ and $A$ are contractible objects. 
Theorem 3.7. Given two contractible cofibrations $A \stackrel{i}{\longleftarrow} B \stackrel{i^{\prime}}{\longmapsto} A^{\prime}$, the pushout object $P\left\{i, i^{\prime}\right\}$ is contractible.

Proof. By the NEP, there are extensions $r: C B \rightarrow B, q^{\prime}: C A^{\prime} \rightarrow A^{\prime}$ and $q: C A \rightarrow A$ of the morphisms $1_{B},\left\{i^{\prime} r, 1_{A^{\prime}}\right\}$ and $\left\{i r, 1_{A}\right\}$ relative to $\kappa_{B}, i_{1}^{\prime}$ and $i_{1}$, respectively. The morphism $q \cup q^{\prime}: P\left\{C i, C i^{\prime}\right\} \rightarrow P\left\{i, i^{\prime}\right\}$ is a retraction of $\kappa_{P\left\{i, i^{\prime}\right\}}=\kappa_{A} \cup \kappa_{A^{\prime}}$.

Observe that, by Theorem 3.7, if $i: B \longmapsto A$ is a contractible cofibration, then the object $\Sigma^{i_{n}}$ and the cofibration $i_{n+1}: \Sigma^{i_{n}} \longmapsto C^{n+1} A$ are also contractible, for every $n \geq 0$.

In homotopy theory the notion of point is generally used to obtain homotopy groups of pointed objects through suspension objects. Next, we define such a notion in a $C$ category.

Definition 3.8. Let $\emptyset$ be the initial object of a C-category. An object $A$ is said to be cofibrant when the initial morphism $\emptyset_{A}: \emptyset \rightarrow A$ is a cofibration.

In $C$-categories where each object is cofibrant, we can omit in C3 the phrase "The morphisms $1_{X}$ and $\kappa_{X}$ are always cofibrations". Note that $1_{X}=\overline{1_{\emptyset}}: X \rightarrow X=P\left\{\emptyset_{X}, 1_{\emptyset}\right\}$ is a cofibration by $\mathbf{C 2}$. On the other hand, as a consequence of $\mathbf{C 2}, \mathbf{C} 3$ and $\mathbf{C 4}$, we have that the inclusion $\kappa_{X}=\left(\emptyset_{X}\right)_{1} \overline{\kappa_{\emptyset}}=\left(\emptyset_{X}\right)_{1} \overline{\emptyset_{C \emptyset}}$ is also a cofibration.

Definition 3.9. A $C$-category is said to be pointed if every object is cofibrant and the cone of the initial object is the initial object.

In pointed categories the initial object is denoted by $*$ and is termed a point. We remark that $* \simeq 0($ since $*=C *)$ and $\left(*_{X}\right)_{1}=\kappa_{X}$.

Given two objects $X$ and $Y$, we will denote by $X \vee Y$ the object $P\left\{*_{X}, *_{Y}\right\}$. By $\mathbf{C 2}$ it is clear that $C(X \vee Y)=C X \vee C Y$. By Remark 3.3, if $i: B \longmapsto A$ and $i^{\prime}: B^{\prime} \longmapsto A^{\prime}$ are cofibrations, then so is $i \vee i^{\prime}: B \vee B^{\prime} \longmapsto A \vee A^{\prime}$. Finally, if $X \simeq 0$ and $Y \simeq 0$, then $X \vee Y \simeq 0$, by Theorem 3.7 .

Next, we introduce the notion of relative homotopy in a (not necessarily pointed) $C$-category, and prove that it is an equivalence relation compatible with the composition of morphisms.

Definition 3.10. Given a cofibration $i: B \longmapsto C A$ and two morphisms $f_{0}, f_{1}: C A \rightarrow X$, the morphism $f_{0}$ is said to be homotopic to the morphism $f_{1}$ relative to $i$ if there exists an extension $F: C^{2} A \rightarrow X$ of the morphism $\left\{f_{0} \rho C i, f_{1}\right\}$ relative to $i_{1}$. The morphism $F$ is called a homotopy from $f_{0}$ to $f_{1}$ relative to $i$, in symbols $F: f_{0} \simeq f_{1}$ rel. $i$.

The existence of the morphism $\left\{f_{0} \rho C i, f_{1}\right\}$ implies that $f_{0} i=f_{1} i$.

Theorem 3.11. The relative homotopy relation is an equivalence relation on the set 
$\operatorname{Hom}(C A, X)^{u(i)}$.

Proof. By the NEP there exists the following extension $\mu$

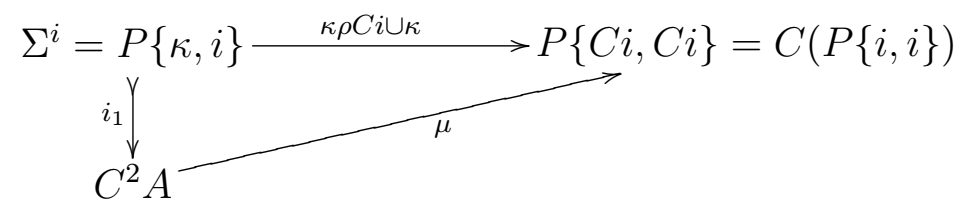

that will be fundamental in this proof and, later, in the construction of the homotopy groups.

Clearly $f \rho: f \simeq f$ rel. $i$.

If $F: f_{0} \simeq f_{1}$ rel. $i$, then $\bar{F}=\left\{F, f_{0} \rho\right\} \mu: f_{1} \simeq f_{0}$ rel. $i$.

Finally, if $F: f_{0} \simeq f_{1}$ and $G: f_{1} \simeq f_{2}$ rel. $i$, then $F \cdot G=\{\bar{F}, G\} \mu: f_{0} \simeq f_{2}$ rel. $i$.

The quotient set $\operatorname{Hom}(C A, X)^{u(i)} / \simeq$ will be denoted by $[C A, X]^{u(i)}$.

Remark 3.12. By the NEP, if $X \simeq 0$, then the set $[C A, X]^{u(i)}$ is always unitary. By Theorem 3.7 given $i: B \longmapsto A$ with $B \simeq 0$, the cofibration $i_{1}$ is contractible and $\left.\left[C A, X^{\prime}\right]\right]^{\prime}(i)$ is always unitary. In sum, if $X$ or $i$ is contractible, then $f_{0} \simeq f_{1}$ rel. $i$ if and only if $f_{0} i=f_{1} i$.

Theorem 3.13. Given a cofibration $i: B \longmapsto C A$, every morphism $f: X \rightarrow Y$ induces a map $f_{*}:[C A, X]^{u(i)} \rightarrow[C A, Y]^{f u(i)}$ defined by $f_{*}([h])=[$ fh $]$. Moreover, every commutative square $C f i=i^{\prime}$ g relating cofibrations $i: B \longmapsto C A$ and $i^{\prime}: B^{\prime} \longmapsto C A^{\prime}$ induces a map $(C f)^{*}:\left[C A^{\prime}, X\right]^{u\left(i^{\prime}\right)} \rightarrow[C A, X]^{u g(i)}$ defined by $(C f)^{*}\left(\left[h^{\prime}\right]\right)=\left[h^{\prime} C f\right]$. If the square is a pushout, then $(C f)^{*}$ is a bijection.

Proof. If $H: h_{0} \simeq h_{1}$ rel. $i$, then $f H: f h_{0} \simeq f h_{1}$ rel. $i$.

If $H^{\prime}: h_{0}^{\prime} \simeq h_{1}^{\prime}$ rel. $i^{\prime}$, then $H^{\prime} C^{2} f: h_{0}^{\prime} C f \simeq h_{1}^{\prime} C f$ rel. $i$.

If the square is a pushout and $H: h_{0} \simeq h_{1}$ rel. $i$, then $\left\{H,\left\{h_{0}, u\right\} \rho C i^{\prime}\right\}: C^{2} A^{\prime}=$ $P\{C i, C f\} \rightarrow X$ makes $\left\{h_{0}, u\right\} \simeq\left\{h_{1}, u\right\}$ rel. $i^{\prime}$.

Finally, we will see the behaviour of the homotopy relative to a coproduct of cofibrations.

Proposition 3.14. Given cofibrations $i: B \longmapsto C A, i^{\prime}: B^{\prime} \longmapsto C A^{\prime}$ and a morphism $\left\{u, u^{\prime}\right\}: B \vee B^{\prime} \rightarrow X$, there exists a bijection

$$
\left[C A \vee C A^{\prime}, X\right]^{\left\{u, u^{\prime}\right\}\left(i \vee i^{\prime}\right)} \cong[C A, X]^{u(i)} \times\left[C A^{\prime}, X\right]^{u^{\prime}\left(i^{\prime}\right)}
$$

Proof. It suffices to observe that $F: f_{0} \simeq f_{1}$ rel. $i$ and $F^{\prime}: f_{0}^{\prime} \simeq f_{1}^{\prime}$ rel. $i^{\prime}$ if and only if $\left\{F, F^{\prime}\right\}:\left\{f_{0}, f_{0}^{\prime}\right\} \simeq\left\{f_{1}, f_{1}^{\prime}\right\}$ rel. $i \vee i^{\prime}$. 


\section{Homotopy groups}

In this section, homotopy groups relative to a cofibration or referred to an object, will be constructed through suspension functors. Also, the usual properties of these homotopy groups will be studied. The relative homotopy relation will be extended to arbitrary cofibrations, whose codomain is not necessarily a cone object.

Throughout this section $\mathbf{C}$ will be a pointed $C$-category. Symbols ${ }^{-}$and $\cdot$ are used here, with the same meaning as in the proof of Theorem 3.11, to define the inverse and composition operations, respectively, in the homotopy groups.

Firstly, we define the category of based objects, where it is possible to define zero morphisms. These concepts permit the definition of homotopy groups.

Definition 4.1. The category of based objects of $\mathbf{C}$ is defined as follows. Objects are pairs $(X, \alpha)$, where $\mathrm{X}$ is an object and $\alpha: X \rightarrow *$ is a morphism of $\mathrm{C}$. A morphism $f:(X, \alpha) \rightarrow(Y, \beta)$ is $f: X \rightarrow Y$ such that $\beta f=\alpha$. If $f: X \longmapsto Y$ is a cofibration in $\mathbf{C}$, then $f:(X, \alpha) \longmapsto(Y, \beta)$ will be called a based cofibration.

Every object of $\mathbf{C}$ has at least a base morphism, since it is cofibrant and $* \simeq 0$.

Definition 4.2. Given a based object $(X, \alpha)$, the zero morphism $0: X \rightarrow Y$ is defined by $0=*_{Y} \alpha$.

The cone functor and the natural transformations $\kappa, \rho$ can be extended to the category of based objects: $C(X, \alpha)=(C X, C \alpha)$ and $C f:(C X, C \alpha) \rightarrow(C Y, C \beta)$ with $\kappa:(X, \alpha) \longmapsto(C X, C \alpha)$ and $\rho:\left(C^{2} X, C^{2} \alpha\right) \rightarrow(C X, C \alpha)$.

On the other hand, given two based morphisms $(Y, \beta) \stackrel{f}{\leftarrow}(X, \alpha) \stackrel{g}{\rightarrow}(Z, \gamma)$, if there exists the pushout object $P\{f, g\}$ in $\mathbf{C}$, then $(P\{f, g\},\{\beta, \gamma\})$ is the pushout object in the category of based objects. In particular, for every based cofibration $i:(B, \beta) \longmapsto(A, \alpha)$ the object $\Sigma^{i_{n}}$ is based on $\left\{C^{n+1} \beta, C^{n} \alpha, \ldots+\ldots, C^{n} \alpha\right\}$, for each natural number $n$.

In this way, the category of based objects verifies the axioms of a pointed $C$-category except the NEP. Note that $(*, 1)$ is the zero object.

The following result is an important tool to define the product on the homotopy groups and prove Theorem 4.7 .

Theorem 4.3. Given a based cofibration $i:(B, \beta) \longmapsto(C A, C \alpha)$, each extension $\mu$ of $\kappa \rho C i \cup \kappa$ relative to $i_{1}$ in $\mathbf{C}$ induces a bijection $\mu^{*}:[P\{C i, C i\}, X]^{0(\kappa)} \rightarrow\left[C^{2} A, X\right]^{0\left(i_{1}\right)}$.

Proof. If $H: F_{0} \simeq F_{1}$ rel. $\kappa$, then $H \nu: F_{0} \mu \simeq F_{1} \mu$ rel. $i_{1}$, where $\nu$ is an extension of $\left\{C \kappa \mu \rho C i_{1}, \kappa \mu\right\}: \Sigma^{i_{1}}=P\left\{\kappa, i_{1}\right\} \rightarrow C^{2} P\{i, i\}$ rel. $i_{2}$. We point out that $\left\{C \kappa \mu \rho C i_{1}, \kappa \mu\right\}$ is nullhomotopic since its codomain is a cone object. Therefore, $\mu^{*}$ is well defined.

The inverse map is defined by $\left(\mu^{*}\right)^{-1}([F])=[\{0, F\}]$.

If $H: F_{0} \simeq F_{1}$ rel. $i_{1}$, then $\{0, H\}:\left\{0, F_{0}\right\} \simeq\left\{0, F_{1}\right\}$ rel. $\kappa$. So, $\left(\mu^{*}\right)^{-1}$ is well 
defined.

$\left(\mu^{*}\right)^{-1} \mu^{*}=1$, since $\{F C \rho,\{F \rho, G \rho\} C \mu\}:\{F, G\} \simeq\{0,\{F, G\} \mu\}$ rel. $\kappa$. $\mu^{*}\left(\mu^{*}\right)^{-1}=1$, since $\{0, F \rho\} C \mu: F \simeq\{0, F\} \mu$ rel. $i_{1}$.

Remark 4.4. If $F: F_{0} \simeq F_{1}$ rel. $i_{1}$ and $G: G_{0} \simeq G_{1}$ rel. $i_{1}$, then $\{F, G\}:\left\{F_{0}, G_{0}\right\} \simeq$ $\left\{F_{1}, G_{1}\right\}$ rel. $\kappa$.

Next, given a based cofibration $i: B \longmapsto C A$ and an object $X$, we define a group structure on $\left[C^{2} A, X\right]^{0\left(i_{1}\right)}$.

Lemma 4.5. For every based cofibration $i: B \longmapsto C A$ and object $X$, there are a symmetric $\operatorname{map}\left[C^{2} A, X\right]^{0\left(i_{1}\right)} \rightarrow\left[C^{2} A, X\right]^{0\left(i_{1}\right)}$ and a product map $\left[C^{2} A, X\right]^{0\left(i_{1}\right)} \times\left[C^{2} A, X\right]^{0\left(i_{1}\right)} \rightarrow$ $\left[C^{2} A, X\right]^{0\left(i_{1}\right)}$ defined by $[F]^{-1}=[\bar{F}]$ and $[F] \cdot[G]=[F \cdot G]$, respectively.

Proof. If $F: F_{0} \simeq F_{1}$ rel. $i_{1}$, then, by Remark 4.4, $\{F, 0\}:\left\{F_{0}, 0\right\} \simeq\left\{F_{1}, 0\right\}$ rel. $\kappa$. Hence, by Theorem 4.3, $\overline{F_{0}}=\left\{F_{0}, 0\right\} \mu \simeq\left\{F_{1}, 0\right\} \mu=\overline{F_{1}}$ rel. $i_{1}$.

Similarly, if $G: G_{0} \simeq G_{1}$ rel. $i_{1}$ and $H: \overline{F_{0}} \simeq \overline{F_{1}}$ rel. $i_{1}$, then $\{H, G\}:\left\{\overline{F_{0}}, G_{0}\right\} \simeq$ $\left\{\overline{F_{1}}, G_{1}\right\}$ rel. $\kappa$. Hence, $F_{0} \cdot G_{0}=\left\{\overline{F_{0}}, G_{0}\right\} \mu \simeq\left\{\overline{F_{1}}, G_{1}\right\} \mu=F_{1} \cdot G_{1}$ rel $i_{1}$.

Remark 4.6. $[0]^{-1}=[\overline{0}]=[\{0,0\} \mu]=[0]$, by Lemma 4.5 and Theorem 4.3.

Theorem 4.7. If $i: B \longmapsto C A$ is a based cofibration, then $\left[C^{2} A, X\right]^{0\left(i_{1}\right)}$ is a group.

\section{Proof.}

1. If $[F] \in\left[C^{2} A, X\right]^{0\left(i_{1}\right)}$, then:

(a) $[F] \cdot[0]=[F]$. It is a consequence of Theorem 4.3 and Lemma 4.5, since $\{\{F \rho, 0\} C \mu, F C \rho\}:\{0, F\} \simeq\{\{F, 0\} \mu, 0\}$ rel. $\kappa$.

(b) $[0] \cdot[F]=[F]$. It is a consequence of Theorem 4.3, Lemma 4.5 and Remarks 4.4 and 4.6, since $[0] \cdot[F]=[0 \cdot F]=[\{\overline{0}, F\} \mu]=[\{0, F\} \mu]=[F]$.

2. If $[F] \in\left[C^{2} A, X\right]^{0\left(i_{1}\right)}$, then:

(a) $[F] \cdot[F]^{-1}=[0]$. It is a consequence of Theorem 4.3 and Lemma 4.5, since $\{\bar{F} C \rho, \bar{F} C \rho\}:\{\bar{F}, \bar{F}\} \simeq\{0,0\}$ rel. $\kappa$.

(b) $[F]^{-1} \cdot[F]=[0]$. It is a consequence of Lemma 4.5, 2(a) and $1(a)$, observing that $\left([F]^{-1}\right)^{-1}=[\bar{F}]^{-1}=[\{\bar{F}, 0\} \mu]=[F \cdot 0]=[F] \cdot[0]=[F]$. Hence, $[F]^{-1} \cdot[F]=$ $[F]^{-1} \cdot\left([F]^{-1}\right)^{-1}=[\bar{F}] \cdot[\bar{F}]^{-1}=[0]$.

3. Finally, if $[F],[G],[H] \in\left[C^{2} A, X\right]^{0\left(i_{1}\right)}$, then $([F] \cdot[G]) \cdot[H]=[F] \cdot([G] \cdot[H])$. It is a consequence of Lemma 4.5 , observing that every $\left[F_{0}\right],\left[F_{1}\right],\left[F_{2}\right] \in\left[C^{2} A, X\right]^{0\left(i_{1}\right)}$ verify:

(i) $\left[F_{0}\right]^{-1} \cdot\left[F_{1}\right]^{-1}=\left(\left[F_{1}\right] \cdot\left[F_{0}\right]\right)^{-1}$ by Theorem 4.3, Remark 4.4 and the observation made in $2(b)$, since $\left\{\left\{\overline{F_{1}} \rho, F_{0} \rho\right\} C \mu, \overline{F_{1}} C \rho\right\}:\left\{F_{0}, \overline{F_{1}}\right\} \simeq\left\{\left\{\overline{F_{1}}, F_{0}\right\} \mu, 0\right\}$ rel. $\kappa$.

(ii) $\left[F_{0}\right] \cdot\left[F_{2}\right]=\left(\left[F_{0}\right] \cdot\left[F_{1}\right]\right) \cdot\left(\left[F_{1}\right]^{-1} \cdot\left[F_{2}\right]\right)$ as a consequence of $(i)$, Theorem 4.3, Remark 4.4, and the observation made in $2(b)$, since $\left\{\left\{F_{1} \rho, \overline{F_{0}} \rho\right\} C \mu,\left\{F_{1} \rho, F_{2} \rho\right\} C \mu\right\}$ : $\left\{\overline{F_{0}}, F_{2}\right\} \simeq\left\{\left\{F_{1}, \overline{F_{0}}\right\} \mu,\left\{F_{1}, F_{2}\right\} \mu\right\}$ rel. $\kappa$. 
(iii) $\left[F_{0}\right]=\left[F_{1}\right]^{-1} \cdot\left(\left[F_{1}\right] \cdot\left[F_{0}\right]\right)$ by $1(b)$ and $(i i)$, since $\left[F_{0}\right]=[0] \cdot\left[F_{0}\right]=\left([0] \cdot\left[F_{1}\right]^{-1}\right)$. $\left(\left[F_{1}\right] \cdot\left[F_{0}\right]\right)=\left[F_{1}\right]^{-1} \cdot\left(\left[F_{1}\right] \cdot\left[F_{0}\right]\right)$.

Hence, $([F] \cdot[G]) \cdot[H]=([F] \cdot[G]) \cdot\left([G]^{-1} \cdot([G] \cdot[H])\right)=[F] \cdot([G] \cdot[H])$.

Remark 4.8. The group structure of $\left[C^{2} A, X\right]^{0\left(i_{1}\right)}$ does not depend on the choice of the extension $\mu$, since $P\{C i, C i\}=C P\{i, i\} \simeq 0$ and two arbitrary extensions of $\kappa \rho C i \cup \kappa$ relative to $i_{1}$ are homotopic relative to the cofibration, by Remark 3.12.

Definition 4.9. The first homotopy group of an object $X$ relative to a based cofibration $i: B \rightarrow C A$ is defined by $\pi_{1}^{i}(X)=\left[C^{2} A, X\right]^{0\left(i_{1}\right)}$. The nth-homotopy group of $X$ relative to $i$ is defined by $\pi_{n}^{i}(X)=\pi_{1}^{i_{n-1}}(X)$.

Given a based object $A$, the group $\pi_{n}^{*_{A}}(X)$ is also denoted by $\pi_{n}^{A}(X)$ and is called the nth-homotopy group of $\mathrm{X}$ referred to $\mathrm{A}$.

Remark 4.10. It is obvious, by Definition 4.9 , that $\pi_{n}^{i}(X)=\pi_{n-s}^{i_{s}}(X)$. In this way, it is possible to define homotopy groups relative to arbitrary cofibrations, whose codomain is not a cone object, for $n \geq 2$.

Remark 4.11. In order to obtain generalized homotopy groups, in the research announcement [4] we give a generalization of the above construction in a not necesarily pointed $C$-category. Given a cofibration $i: B \longmapsto C A$ and an object $X$, we define a groupoid whose objects are the elements of $\operatorname{Hom}(C A, X)$. Morphisms from $f_{0}$ to $f_{1}$ are homotopy classes relative to $i_{1}$ of homotopies from $f_{0}$ to $f_{1}$. Identities are $1_{f}=[f p]$, inverse morphisms are $[F]^{-1}=[\bar{F}]$ and composite morphisms are $[F] \cdot[G]=[F * G]$. We believe that this groupoid allows one to reconstruct a track category in the sense of H.J. Baues (see [1]). These ideas will be developed in full detail in a later paper.

The following theorem shows the functorial character of the homotopy groups. First, we prove a preliminary result.

Lemma 4.12. Every based commutative square $f i=i^{\prime} g$ relating based cofibrations $i$ : $B \longmapsto A$ and $i^{\prime}: B^{\prime} \longmapsto A^{\prime}$ generates, for each natural number $n$, a based commutative square $C^{n} f i_{n}=i_{n}^{\prime} \Sigma_{n}(f, g)$ relating the based cofibrations $i_{n}: \Sigma^{i_{n-1}} \longmapsto C^{n} A$ and $i_{n}^{\prime}$ : $\Sigma^{i^{\prime}}{ }_{n-1} \longmapsto C^{n} A^{\prime}$. If the first square $f i=i^{\prime} g$ is a pushout, then the generated squares $C^{n} f i_{n}=i_{n}^{\prime} \Sigma_{n}(f, g)$ are pushouts.

Proof. Clearly $i_{1}^{\prime} \Sigma_{1}(f, g)=C f i_{1}$. On the other hand, if the square $f i=i^{\prime} g$ is a pushout and $u: C A \rightarrow X, v=\left\{v_{0}, v_{1}\right\}: \Sigma^{i^{\prime}} \rightarrow X$ verify $u i_{1}=v \Sigma_{1}(f, g)$, then $u C i=v_{0} C g$, and by C2 there exists $\left\{u, v_{0}\right\}: C A^{\prime} \rightarrow X$. Hence, $\left\{u, v_{0}\right\}=\{u, v\}: C A^{\prime}=P\left\{i_{1}, \Sigma_{1}(f, g)\right\} \rightarrow$ $X$. By induction, since $i_{n}=\left(i_{n-1}\right)_{1}$, the same proof is valid.

Theorem 4.13. Given a based cofibration $i: B \longmapsto A$, every morphism $f: X \rightarrow Y$ 
induces homomorphisms of groups $f_{*}: \pi_{n}^{i}(X) \rightarrow \pi_{n}^{i}(Y)$. Moreover, every based commutative square $f i=i^{\prime} g$ relating based cofibrations $i: B \longmapsto A$ and $i^{\prime}: B^{\prime} \longmapsto A^{\prime}$ induces, for each object $X$ and $n \geq 2$, a homomorphism of groups $\left(C^{n} f\right)^{*}: \pi_{n}^{i^{\prime}}(X) \rightarrow \pi_{n}^{i}(X)$. If the square is a push out then $\left(C^{n} f\right)^{*}$ is an isomorphism.

Proof. By the first part of Theorem $3.13 f_{*}$ is a map. Moreover, $f_{*}$ is a homomorphism of groups since $f_{*}([F] \cdot[G])=f_{*}([\{\{F, 0\} \mu, G\} \mu])=([\{\{f F, 0\} \mu, f G\} \mu])=[f F] \cdot[f G]=$ $f_{*}([F]) \cdot f_{*}([G])$.

By the first part of Lemma 4.12 and the second part of Theorem $3.13\left(C^{n} f\right)^{*}$ is a map. If $\mu_{n}$ and $\mu_{n}^{\prime}$ are extensions used to obtain the operations in $\pi_{n}^{i}(X)$ and $\pi_{n}^{i^{\prime}}(X)$, respectively, then $\mu_{n}^{\prime} C^{n} f i_{n}=\left(C^{n} f \cup C^{n} f\right) \mu_{n} i_{n}$. Hence, $\mu_{n}^{\prime} C^{n} f \simeq\left(C^{n} f \cup C^{n} f\right) \mu_{n}$ rel. $i_{n}$ by Remark 3.12, and $\left(C^{n} f\right)^{*}([F] \cdot[G])=\left(C^{n} f\right)^{*}([F]) \cdot\left(C^{n} f\right)^{*}([G])$.

The proof is concluded by the second part of Lemma 4.12 and the third part of Theorem 3.13.

Remark 4.14. Isomorphisms of groups induced by $f$ in the second part of Theorem 4.13 allow us to define homotopy relative to cofibrations whose codomain is not just a cone object. If $A$ or $A^{\prime}$ is a cone object, then there is homotopy relative to $i$ or $i^{\prime}$, respectively. Since the sets $[C A, X]^{0\left(i_{1}\right)}$ and $\left[C A^{\prime}, X\right]^{0\left(i_{1}^{\prime}\right)}$ are bijective and the groups $\pi_{n}^{i}(X)$ and $\pi_{n}^{i^{\prime}}(X)$ are isomorphic for $n \geq 2$, it seems natural to translate the group structure via the bijection, obtaining in this way the isomorphism of groups for $n=1$. Moreover, we can assume $[A, X]^{u g(i)} \cong\left[A^{\prime}, X\right]^{u\left(i^{\prime}\right)}$, that is, $f_{0} \simeq f_{1}$ rel. $i^{\prime}$ if and only if $f_{0} f \simeq f_{1} f$ rel. $i$. Repeating this process, one can obtain homotopy relative to cofibrations whose codomain is not a cone object, through another such cofibration. When the cofibration is the initial morphism, then $[A, X]^{*_{X}\left(*_{A}\right)}$ is simply denoted by $[A, X]$.

As a consequence of this last convention, the homotopy groups relative to a cofibration or referred to an object can be obtained through suspension objects.

Definition 4.15. Given a based cofibration $i:(B, \beta) \longmapsto(C A, C \alpha)$, the suspension object of $i$ is defined by $S_{i}=P\{i, \beta\}$. Given a based object $(A, \alpha)$, the n-suspension object of $(A, \alpha)$ is inductively defined by $S^{n} A=S_{\kappa_{S^{n-1} A}}$, with $S^{0} A=A$. The notation $S_{i}^{n}$ will designate the object $S^{n-1} S_{i}$.

Lemma 4.16. If $(A, \alpha)$ is a based object such that $A=P\{j, s\}$, where $j$ is a cofibration and the codomain of the morphism $s$ is the object $*$, then $S A=P\left\{j_{1},\{C s, \alpha \bar{s}\}\right\}$. 
Proof. Since $\alpha\{\bar{j} C s, \bar{s}\}=\{C s, \alpha \bar{s}\}$, it suffices to observe the following pushouts:

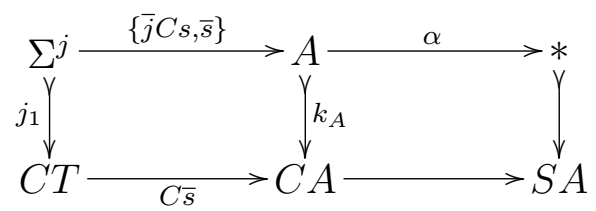

Theorem 4.17. $S_{i}^{n+1}=P\left\{i_{n},\left\{C^{n} \beta, C^{n-1} \alpha, \ldots\left(n, C^{n} \alpha\right\}\right\}\right.$ for every based cofibration $i$ : $(B, \beta) \longmapsto(C A, C \alpha)$ and natural number $n$.

Proof. It is a consequence of the inductive use of the previous Lemma 4.16, since $S_{i}=$ $P\{i, \beta\}, i_{n}=\left(i_{n-1}\right)_{1}$ and $S_{i}^{n+1}=S S_{i}^{n}$.

Corollary 4.18. There are bijections $\pi_{n}^{i}(X) \cong\left[S_{i}^{n+1}, X\right]$ and $\pi_{n+1}^{D}(X) \cong\left[S^{n+1} D, X\right]$ for every based cofibration $i: B \longmapsto C A$, based object $D$ and natural number $n$.

The homotopy groups of this algebraic homotopy theory verify the usual properties of homotopy groups. In this sense, by Remark 3.12, we have:

Proposition 4.19. Given a based cofibration $i: B \longmapsto C A$, a based object $D$, an object $X$ and a natural number $n$ :

a) If $i$ is a contractible cofibration, then $\pi_{n}^{i}(X) \cong\{0\}$.

b) If $D$ is a contractible object, then $\pi_{n+1}^{D}(X) \cong\{0\}$.

c) If $X$ is a contractible object, then $\pi_{n}^{i}(X) \cong \pi_{n+1}^{D}(X) \cong\{0\}$.

The following properties emerge as particular cases of Remark 4.10 and Theorem 4.13. We point out that, by Lemma 4.16, the suspensions of every based object $A$ verify $S^{n} A=S^{n-r} S^{r} A$, for $0 \leq r \leq n$.

Proposition 4.20. Given a based object D, a morphism $f: X \rightarrow Y$ and a based morphism $g: A \rightarrow B$ :

a) The homotopy groups $\pi_{n}^{A}(X)$ and $\pi_{n-r}^{S^{r}}(X)$ are isomorphic.

b) The map $f_{*}: \pi_{n}^{A}(X) \rightarrow \pi_{n}^{A}(Y)$ is a homomorphism of groups.

c) The map $\left(C^{n} g\right)^{*}: \pi_{n}^{B}(X) \rightarrow \pi_{n}^{A}(X)$ is a homomorphism of groups.

d) If $g$ is an isomorphism, then $\left(C^{n} g\right)^{*}$ is an isomorphism of groups.

Homotopy groups relative to a coproduct are products of homotopy groups.

Proposition 4.21. Given two based cofibrations $i: B \longmapsto C A$ and $i^{\prime}: B^{\prime} \longmapsto C A^{\prime}$, two based objects $D$ and $D^{\prime}$, and an object $X$ :

a) The groups $\pi_{n}^{i \vee i^{\prime}}(X)$ and $\pi_{n}^{i}(X) \times \pi_{n}^{i^{\prime}}(X)$ are isomorphic.

b) The groups $\pi_{n}^{D \vee D^{\prime}}(X)$ and $\pi_{n}^{D}(X) \times \pi_{n}^{D^{\prime}}(X)$ are isomorphic. 
Proof. It is easily seen by Proposition 3.14 and Remark 4.8, since the coproduct preserves pushouts and $\mu_{n} \vee \mu_{n}^{\prime}$ extends $\left(\kappa \rho C i_{n-1} \vee \kappa \rho C i_{n-1}^{\prime}\right) \cup(\kappa \vee \kappa)$ rel. $i_{n} \vee i_{n}^{\prime}$.

\section{$5 \quad$ Exact sequences of homotopy groups}

In homotopy theory, homotopy groups are usually related through exact sequences. In $C$-categories, this relationship is also possible.

In the classical sense, a pair $(X, Y)$ is a cofibration $f: Y \longmapsto X$. The category of such pairs in a $C$-category is also a $C$-category. Therefore, the development made for a $C$-category is also available for its category of pairs.

Definition 5.1. Given a $C$-category $\mathbf{C}$, the category cof $\mathbf{C}$ is the full subcategory of Pair $\mathbf{C}$ whose objects are the cofibrations of $\mathbf{C}$.

The pairs $(X, Y),\left(X^{\prime}, Y^{\prime}\right), \ldots$ will denote, respectively, the objects $f: Y \succ X, f^{\prime}$ : $Y^{\prime} \longmapsto X^{\prime}, \ldots$ in cof $\mathbf{C}$. Given an object $A$ in $\mathbf{C}, 1_{A}$ and $\kappa_{A}$ are the cofibrations associated to the pairs $(A, A)$ and $(C A, A)$, respectively.

Definition 5.2. A morphism $(u, v):(X, Y) \longmapsto\left(X^{\prime}, Y^{\prime}\right)$ in cof $\mathbf{C}$ is a cofibration of pairs if $v: Y \longmapsto Y^{\prime}$ and $\left\{f^{\prime}, u\right\}: P\{v, f\} \longmapsto X^{\prime}$ are cofibrations in C.

We point out that if $(u, v)$ is a cofibration, then so is $u=\left\{f^{\prime}, u\right\} \bar{v}$.

Theorem 5.3. If $\mathbf{C}$ is a $C$-category, then so is $\mathbf{c o f} \mathbf{C}$, with the cofibrations of pairs.

Proof. The cone functor is defined for objects by $C(X, Y)=(C X, C Y)$, with associated cofibration $C f$, and for morphisms by $C(g, h)=(C g, C h)$. The natural transformations $\kappa$ and $\rho$ are defined by $(\kappa, \kappa)$ and $(\rho, \rho)$, respectively.

- The cone axiom is clearly satisfied.

- Pushout axiom: Given $\left(X^{\prime}, Y^{\prime}\right) \stackrel{(u, v)}{\longleftrightarrow}(X, Y) \stackrel{(g, h)}{\rightarrow}\left(X^{\prime \prime}, Y^{\prime \prime}\right)$, then $P\{(u, v),(g, h)\}=$ $(P\{u, g\}, P\{v, h\})$ with associated cofibration $f^{\prime} \cup f^{\prime \prime}$. The pushouts $P\{u, g\}$ and $P\{v, h\}$ exist by $\mathbf{C} \mathbf{2}$ in $\mathbf{C}$, and $f^{\prime} \cup f^{\prime \prime}$ is a cofibration in $\mathbf{C}$ by Remark 3.3.

On the other hand, given the following diagram

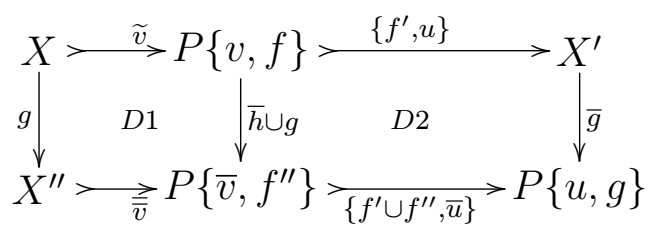

where $\bar{v}: Y^{\prime \prime} \longmapsto P\{v, h\}$. The commutative square $D 1$ is composable with the pushout of $P\{v, f\}$, and the composite diagram is the pushout of $P\left\{v, f^{\prime \prime} h\right\}$. Therefore, $D 1$ is a pushout, and so is $D 2$, since the composite diagram is the pushout of $P\{u, g\}$. So that, $\left\{f^{\prime} \cup f^{\prime \prime}, \bar{u}\right\}=\overline{\left\{f^{\prime}, u\right\}}: P\left\{\bar{v}, f^{\prime \prime}\right\} \longmapsto P\{u, g\}$ is a cofibration, and 
$(\bar{u}, \bar{v})$ is a cofibration of pairs.

Clearly, the functor $C$ carries cofibrated pushouts to push outs.

- Cofibration axiom: The morphisms $1_{(X, Y)}$ and $\kappa_{(X, Y)}$ are always cofibrations, since $\left\{f, 1_{X}\right\}=1_{X}: P\left\{1_{Y}, f\right\}=X \longmapsto X$ and $\left\{C f, \kappa_{X}\right\}=f_{1}: P\left\{\kappa_{Y}, f\right\}=\Sigma^{f} \longmapsto C X$ are cofibrations in $\mathbf{C}$, by $\mathbf{C} 3$ and $\mathbf{C 4}$, respectively.

Given $(X, Y) \stackrel{(u, v)}{\longmapsto}\left(X^{\prime}, Y^{\prime}\right) \stackrel{\left(u^{\prime}, v^{\prime}\right)}{\longmapsto}\left(X^{\prime \prime}, Y^{\prime \prime}\right)$, the composite $\left(u^{\prime} u, v^{\prime} v\right)$ is a cofibration, since $\left\{f^{\prime \prime}, u^{\prime} u\right\}=\left\{f^{\prime \prime}, u^{\prime}\right\}(1 \cup u): P\left\{v^{\prime} v, f\right\} \longmapsto P\left\{v^{\prime}, f^{\prime}\right\} \longmapsto X^{\prime \prime}$ is a cofibration in C. Observe that $1 \cup u$ is a cofibration by Remark 3.3.

Every cofibration $(u, v):(X, Y) \longmapsto\left(X^{\prime}, Y^{\prime}\right)$ verifies the nullhomotopy extension property. By the NEP in $\mathbf{C}$, there is a retraction $r_{v}$ for $C v$. By Theorem 3.5, there is an extension $r_{u}$ of the morphism $\left\{C f r_{v}, 1_{C X}\right\}$ relative to the cofibration $\left\{C f^{\prime}, C u\right\}=$ $C\left\{f^{\prime}, u\right\}$. The morphism of pairs $\left(r_{u}, r_{v}\right)$ is a retraction for $(C u, C v)=C(u, v)$.

- Relative cone axiom: If $(u, v):(X, Y) \longmapsto\left(X^{\prime}, Y^{\prime}\right)$ is a cofibration, then so is $(u, v)_{1}=$ $\left(u_{1}, v_{1}\right): \Sigma^{(u, v)}=\left(\Sigma^{u}, \Sigma^{v}\right) \longmapsto C\left(X^{\prime}, Y^{\prime}\right)=\left(C X^{\prime}, C Y^{\prime}\right)$, since $P\left\{v_{1}, \Sigma_{1}\left(f^{\prime}, f\right)\right\}=$ $\Sigma^{\left\{f^{\prime}, u\right\}}$ with induced morphisms $\overline{\Sigma_{1}\left(f^{\prime}, f\right)}=\overline{\left\{f^{\prime}, u\right\}} C \bar{f}$ and $\overline{v_{1}}=\left\{\overline{\left\{f^{\prime}, u\right\}} C \bar{v}, \bar{\kappa}\right\}$. Hence, $\left\{C f^{\prime},\{C u, \kappa\}\right\}=\left\{f^{\prime}, u\right\}_{1}: P\left\{v_{1}, \Sigma_{1}\left(f^{\prime}, f\right)\right\}=\Sigma^{\left\{f^{\prime}, u\right\}} \longmapsto C X^{\prime}$. By Remark 3.3, $\left(\Sigma^{u}, \Sigma^{v}\right)$ is an object of cof $\mathbf{C}$ with associated cofibration $\Sigma_{1}\left(f^{\prime}, f\right)$, since $f_{1}=$ $\left\{C f, \kappa_{X}\right\}$ is a cofibration.

The cone structure on cof $\mathbf{C}$ arises from the respective structure on $\mathbf{C}$. Therefore, we can relate the homotopy concepts induced by the cone structure in both categories.

Clearly, if $(\widetilde{g}, \widetilde{h})$ is an extension of $(g, h):(X, Y) \rightarrow\left(X^{\prime}, Y^{\prime}\right)$ relative to $(u, v)$ : $(X, Y) \longmapsto\left(X^{\prime \prime}, Y^{\prime \prime}\right)$, then $\widetilde{g}$ and $\widetilde{h}$ are extensions of $g$ and $h$ relative to $u$ and $v$, respectively. In particular, every nullhomotopy of pairs is a pair of nullhomotopies.

It is easily seen that the following equality holds:

$$
\begin{gathered}
\operatorname{Hom}\left(\left(\mathrm{X}^{\prime \prime}, \mathrm{Y}^{\prime \prime}\right),\left(\mathrm{X}^{\prime}, \mathrm{Y}^{\prime}\right)\right)^{(g, h)((u, v))}=\bigcup_{\widetilde{h} \in \operatorname{Hom}\left(Y^{\prime \prime}, Y^{\prime}\right)^{h(v)}} \operatorname{Hom}\left(X^{\prime \prime}, X^{\prime}\right)^{\left\{f^{\prime} \tilde{h}, g\right\}\left(\left\{f^{\prime \prime}, u\right\}\right)} \times\{\widetilde{h}\}
\end{gathered}
$$

Remark 5.4. If $(u, v):(X, Y) \longmapsto\left(C X^{\prime}, C Y^{\prime}\right)$ is a cofibration and $(G, H):\left(g_{0}, h_{0}\right) \simeq$ $\left(g_{1}, h_{1}\right)$ rel. $(u, v)$, then $G: g_{0} \simeq g_{1}$ rel. $u$ and $H: h_{0} \simeq h_{1}$ rel. $v$, since $(u, v)_{1}=\left(u_{1}, v_{1}\right)$. On the other hand, $[(F, G)] \cdot\left[\left(F^{\prime}, G^{\prime}\right)\right]=\left[\left(F \cdot F^{\prime}, G \cdot G^{\prime}\right)\right]$ for every $[(F, G)],\left[\left(F^{\prime}, G^{\prime}\right)\right] \in$ $\pi_{n}^{(u, v)}\left(\left(X^{\prime \prime}, Y^{\prime \prime}\right)\right)$, since an extension of pairs is a pair of extensions, and the operation of the groups in $\mathbf{C}$ does not depend on the choice of the extension (Remark 4.8).

In this way, if the cofibration of pairs $(u, v)$ is based, then the projections $p_{1}$ : $\pi_{n}^{(u, v)}\left(\left(X^{\prime \prime}, Y^{\prime \prime}\right)\right) \rightarrow \pi_{n}^{v}\left(Y^{\prime \prime}\right)$ and $p_{2}: \pi_{n}^{(u, v)}\left(\left(X^{\prime \prime}, Y^{\prime \prime}\right)\right) \rightarrow \pi_{n}^{u}\left(X^{\prime \prime}\right)$, defined by $p_{1}([(F, G)])=$ $[G]$ and $p_{2}([(F, G)])=[F]$, are homomorphisms of groups for every pair $\left(X^{\prime \prime}, Y^{\prime \prime}\right)$.

Some curious properties appear when the codomain of a pair is a contractible object.

Proposition 5.5. Given $\left(X^{\prime \prime}, Y^{\prime \prime}\right) \stackrel{(u, v)}{\longleftarrow}(X, Y) \stackrel{(g, h)}{\longrightarrow}\left(X^{\prime}, Y^{\prime}\right)$, if $X^{\prime}$ is contractible, then $\operatorname{Hom}\left(Y^{\prime \prime}, Y^{\prime}\right)^{h(v)} \neq \emptyset$ if and only if $\operatorname{Hom}\left(\left(X^{\prime \prime}, Y^{\prime \prime}\right),\left(X^{\prime}, Y^{\prime}\right)\right)^{(g, h)((u, v))} \neq \emptyset$. 
Proof. By the equality seen before Remark 5.4 and the NEP and using the fact that $X^{\prime} \simeq 0$, it is seen that for every morphism $\widetilde{h} \in \operatorname{Hom}\left(Y^{\prime \prime}, Y^{\prime}\right)^{h(v)}$ there is a morphism $\widetilde{g} \in \operatorname{Hom}\left(X^{\prime \prime}, X^{\prime}\right)^{\left\{f^{\prime} \tilde{h}, g\right\}\left(\left\{f^{\prime \prime}, u\right\}\right)}$.

Corollary 5.6. In the $C$-category cof $\mathbf{C}$ the following equivalences are verified.

a) Given $(g, h):(X, Y) \rightarrow\left(X^{\prime}, Y^{\prime}\right)$ with $X^{\prime} \simeq 0$, then $(g, h) \simeq 0$ if and only if $h \simeq 0$.

b) $(X, Y) \simeq 0$ if and only if $X \simeq 0$ and $Y \simeq 0$.

c) Given $\left(C X^{\prime \prime}, C Y^{\prime \prime}\right) \stackrel{(u, v)}{\longleftarrow}(X, Y) \stackrel{\left(g_{0}, h_{0}\right),\left(g_{1}, h_{1}\right)}{\longrightarrow}\left(X^{\prime}, Y^{\prime}\right)$, if $X^{\prime} \simeq 0$, then $\left(g_{0}, h_{0}\right) \simeq$ $\left(g_{1}, h_{1}\right)$ rel. $(u, v)$ if and only if $g_{0} \simeq g_{1}$ rel. $u$ and $h_{0} \simeq h_{1}$ rel. $v$.

d) Given $\left(C X^{\prime \prime}, C Y^{\prime \prime}\right) \stackrel{(u, v)}{\longleftarrow}(X, Y) \stackrel{(g, h)}{\longrightarrow}\left(X^{\prime}, Y^{\prime}\right)$, if $X^{\prime} \simeq 0$, then

$$
\left[\left(C X^{\prime \prime}, C Y^{\prime \prime}\right),\left(X^{\prime}, Y^{\prime}\right)\right]^{(g, h)((u, v))} \cong\left[C Y^{\prime \prime}, Y^{\prime}\right]^{h(v)}
$$

A pointed C-category $\mathbf{C}$ induces a pointed structure on cof $\mathbf{C}$.

Proposition 5.7. The category cof $\mathbf{C}$ as an initial object if and only if so has $\mathbf{C}$.

Proof. If $\emptyset$ is initial in $\mathbf{C}$, then $(\emptyset, \emptyset)$ is initial in cof $\mathbf{C}$.

Conversely, if $(X, Y)$ is initial in cof $\mathbf{C}$, then there is an unique morphism $(g, h)$ : $(X, Y) \rightarrow(Y, Y)$. Hence, $(f g, h):(X, Y) \rightarrow(X, Y)$ is the initial morphism $\left(1_{X}, 1_{Y}\right)$. Therefore, $f g=1_{X}$ and $1_{Y}=h=g f$, and so that $(X, X) \cong(X, Y)$ is an initial object. One readily checks that $X$ is initial in $\mathbf{C}$, since this category can be considered as a subcategory of cof $\mathbf{C}$ identifying in a natural way objects $Z$ with $(Z, Z)$.

Using the previous Proposition 5.7, it is clear that $(X, Y)$ is cofibrant if and only if so are $X$ and $Y$. Moreover, $\mathbf{C}$ is pointed if and only if so is cof $\mathbf{C}$.

The pair $(X, Y)$ is based on $(\alpha, \beta)$ if and only if $f:(Y, \beta) \longmapsto(X, \alpha)$ is a based cofibration. Moreover, $(u, v):(X, Y) \longmapsto\left(X^{\prime}, Y^{\prime}\right)$ is based if and only if so are $u$ and $v$.

The part $(d)$ of Corollary 5.6 induces the following isomorphisms of groups.

Corollary 5.8. Given a based cofibration $(u, v):(X, Y) \longmapsto\left(X^{\prime}, Y^{\prime}\right)$, a based object $\left(X^{\prime \prime}, Y^{\prime \prime}\right)$ and an object $\left(X^{\prime \prime \prime}, Y^{\prime \prime \prime}\right)$, if $X^{\prime \prime \prime} \simeq 0$, then $\pi_{n}^{(u, v)}\left(X^{\prime \prime \prime}, Y^{\prime \prime \prime}\right) \cong \pi_{n}^{v}\left(Y^{\prime \prime \prime}\right)$ and $\pi_{n}^{\left(X^{\prime \prime}, Y^{\prime \prime}\right)}\left(X^{\prime \prime \prime}, Y^{\prime \prime \prime}\right) \cong \pi_{n}^{Y^{\prime \prime}}\left(Y^{\prime \prime \prime}\right)$.

Homotopy groups of pairs will be used to build exact homotopy sequences. Next, we introduce important tools to prove this fact.

Proposition 5.9. Given a based cofibration $i: B \longmapsto A$, for every $n \in \mathbb{N}$ there is an isomorphism of groups $\theta: \pi_{n+1}^{\left(i_{1}, 1\right)}((X, Y)) \cong \pi_{n+2}^{i}(X)$, where $\left(i_{1}, 1\right):\left(\Sigma^{i}, A\right) \longmapsto(C A, A)$.

Proof. If $[(F, G)] \in \pi_{n+1}^{\left(i_{1}, 1\right)}((X, Y))$, then $(F, G)\left(i_{n+2}, 1\right)=(0,0)$, so $G=0$. Therefore, by Remark 5.4, $[(F, 0)] \leftrightarrow[F]$ is an isomorphism of groups. 
Definition 5.10. The $(n+1)$-th homotopy group of a pair $(X, Y)$ relative to a based cofibration $i: B \longmapsto A$ is defined by $\pi_{n+1}^{i}((X, Y))=\pi_{n}^{(C i, i)}((X, Y))$.

If $i: B \longmapsto A$ is a based cofibration, then so are $C i: C B \longmapsto C A$ and $(C i, i)$ : $(C B, B) \longmapsto(C A, A)$. On the other hand, if $i: B \longmapsto A$ is a contractible cofibration, then so is $(C i, i):(C B, B) \longmapsto(C A, A)$, and $\pi_{n+1}^{i}((X, Y)) \cong\{0\}$. By Corollary 5.8 , if $X \simeq 0$, then $\pi_{n+1}^{i}((X, Y)) \cong \pi_{n}^{i}(Y)$.

Lemma 5.11. For every based cofibration $i: B \longmapsto A$ and morphism $F: C^{n+1} A \rightarrow X$ verifying $F i_{n+1}=0$, there is an extension of the morphism $\{0, F, 0, \ldots, 0, F, 0\}$ (resp. $\{0, F, 0, \ldots, 0, F\})$ relative to $i_{n+2}$, for every odd (resp. even) natural number $n$.

Proof. If $n$ is odd, then $H C^{\frac{n+3}{2}} \kappa$ extends $\{0, F, 0, \ldots, 0, F, 0\}$ rel. $i_{n+2}$, where $H$ is an extension of $\left\{0, F C^{\frac{n+1}{2}} \rho, F C^{\frac{n-1}{2}} \rho, F C^{\frac{n-3}{2}} \rho, \ldots, F C \rho, F C^{n} \rho, F C^{n-1} \rho, . ., F C^{\frac{n+1}{2}} \rho, 0\right\}$ rel. $\left(C i_{\frac{n+1}{2}}\right)_{\frac{n+3}{2}}$.

If $n$ is even, then $H C^{\frac{n+2}{2}} \kappa$ extends $\{0, F, 0, \ldots, 0, F\}$ rel. $i_{n+2}$, where $H$ is an extension of $\left\{0, F C^{\frac{n}{2}} \rho, F C^{\frac{n-2}{2}} \rho, F C^{\frac{n-4}{2}} \rho, \ldots, F \rho, F C^{n} \rho, F C^{n-1} \rho, \ldots, F C^{\frac{n}{2}} \rho\right\}$ rel. $\left(C i_{\frac{n+2}{2}}\right)_{\frac{n+2}{2}}$.

The existence of the morphisms extended by $H$ follows from Remark 3.2.

Lemma 5.12. Given a based cofibration $i: B \longmapsto A$ and $[F],[G] \in \pi_{n+2}^{i}(X)$, then $[F]=$ $[G]$ if and only if there exists an extension of the morphism $\{0, G, 0, \ldots, 0, F, 0\}$ (resp. $\{0, G, 0, \ldots, 0, F\})$ relative to $i_{n+3}$, for every even (resp. odd) natural number $n$.

Proof. By Lemma 5.11 there is an extension $H$ of the morphism $\{0, F, 0, \ldots, 0, F, 0\}$ (resp. $\{0, F, 0, \ldots, 0, F\})$ rel. $i_{n+3}$, for every even (resp. odd) natural number $n$. If $[F]=[G]$, then there is $K: G \simeq F$ (resp. $K: F \simeq G$ ) rel. $i_{n+2}$. Therefore, $E C \kappa($ resp. $E \kappa)$ is an extension of $\{0, G, 0, \ldots, 0, F, 0\}$ (resp. $\{0, G, 0, \ldots, 0, F\})$ rel. $i_{n+3}$, where $E$ is an extension of $\{0, K, 0, \ldots, 0, F \rho, H\}$ (resp. $\{0, K, 0, \ldots, 0, H\})$ rel. $\left(C i_{n+2}\right)_{1}$ (resp. $\left.C i_{n+3}\right)$.

Conversely, if $n$ is odd and $H_{0}$ is an extension of $\{0, G, 0, \ldots, 0, F\}$ rel. $i_{n+3}$, then $G_{1} C^{n+3} \kappa$ is an extension of $\{0,0, G, 0, \ldots, 0, F, 0\}$ rel. $i_{n+3}$, where $G_{1}$ is an extension of $\left\{0, F \rho, H_{0}, 0, \ldots, 0, F C^{n+1} \rho, F C^{n} \rho\right\}$ rel. $(C i)_{n+3}$.

If $H_{1}=G_{1} C^{n+3} \kappa$, then $G_{2} C^{n+2} \kappa$ is an extension of $\{0,0,0, G, 0, \ldots, 0, F\}$ rel. $i_{n+3}$, where $G_{2}$ is an extension of $\left\{0,0, F \rho, H_{1}, 0, \ldots, 0, F C^{n-1} \rho, F C^{n} \rho\right\}$ rel. $\left(C i_{1}\right)_{n+2}$.

If $H_{2}=G_{2} C^{n+2} \kappa$, then $G_{3} C^{n+1} \kappa$ is an extension of $\{0,0,0,0, G, 0, \ldots, 0, F, 0\}$ rel. $i_{n+3}$, where $G_{3}$ is an extension of $\left\{0,0,0, F \rho, H_{2}, 0, \ldots, 0, F C^{n-1} \rho, F C^{n-2} \rho\right\}$ rel. $\left(C i_{2}\right)_{n+1}$.

This process can be iterated to obtain an extension $H_{n}$ of $\{0, \ldots, 0, G, F, 0\}$ rel. $i_{n+3}$.

The morphism $H_{n+1}=G_{n+1} C^{3} \kappa$ extends $\{0, . ., 0, G, F\}$ rel. $i_{n+3}$, where $G_{n+1}$ is an extension of $\left\{0, \ldots, 0, F \rho, H_{n}, F C \rho\right\}$ rel. $\left(C i_{n}\right)_{3}$. Hence, $H_{n+1}: G \simeq F$ rel. $i_{n+2}$.

Observe that the morphisms extended by $E, G_{1}, G_{2}, \ldots, G_{n+1}$ exist by Remark 3.2.

An analogous process for an extension $H_{0}$ of the morphism $\{0, G, 0, \ldots, 0, F, 0\}$ rel. $i_{n+3}$ gives $H_{n+1} C^{3} \kappa: G \simeq F$ rel. $i_{n+2}$, when $n$ is even.

Finally, we define the exact sequence of homotopy groups associated with a based 
cofibration and a pair.

Theorem 5.13. For every based cofibration $i: B \longmapsto A$ and pair $(X, Y)$, there is an exact sequence of homotopy groups:

$$
\ldots \rightarrow \pi_{3}^{i}(Y) \stackrel{f_{*}}{\rightarrow} \pi_{3}^{i}(X) \stackrel{j}{\rightarrow} \pi_{3}^{i}((X, Y)) \stackrel{\delta}{\rightarrow} \pi_{2}^{i}(Y) \stackrel{f_{*}}{\rightarrow} \pi_{2}^{i}(X)
$$

Proof. The maps $f_{*}$ and $\delta=p_{1}: \pi_{n+2}^{i}((X, Y))=\pi_{n+1}^{(C i, i)}((X, Y)) \rightarrow \pi_{n+1}^{i}(Y)$ are homomorphisms of groups, by the first part of Theorem 4.13 and Remark 5.4 respectively. The maps $j=(1,1)^{*} \theta^{-1}: \pi_{n+2}^{i}(X) \rightarrow \pi_{n+1}^{\left(i_{1}, 1\right)}((X, Y)) \rightarrow \pi_{n+2}^{i}((X, Y))=\pi_{n+1}^{(C i, i)}((X, Y))$ are homomorphisms of groups, by Proposition 5.9 and the second part of Theorem 4.13 applied to the commutative square $(1,1)(C i, i)=\left(i_{1}, 1\right)(\bar{i}, i)$ in cof $\mathbf{C}$, where $\bar{i}$ is the induced cofibration in the pushout of the relative cone $\Sigma^{i}$.

If $[(F, G)] \in \pi_{n+2}^{i}((X, Y))$, then $f_{*} \delta([(F, G)])=f_{*}([G])=[f G]=[0]$, since $H C^{n+2} \kappa$ : $0 \simeq f G$ rel. $i_{n+1}$, where $H$ is an extension of $\{0, \ldots, 0, F\}$ rel. $(C i)_{n+2}$.

If $[F] \in \pi_{n+2}^{i}(X)$, then $\delta j([F])=\delta([(F, 0)])=[0]$.

If $[F] \in \pi_{n+2}^{i}(Y)$, then $j f_{*}([F])=j([f F])=[(f F, 0)]=[(0,0)]$, since $(H, F)$ : $(f F, 0) \simeq(0,0)(\operatorname{resp} .(H, F):(0,0) \simeq(f F, 0))$ rel. $(C i, i)_{n+1}$ where, by Lemma 5.11, $H: C^{n+2} A \rightarrow X$ is an extension of $\{0, F, 0, \ldots, 0, F, 0\}$ (resp. $\{0, F, 0, \ldots, 0, F\}$ ) rel. $i_{n+3}$, when $n$ is even (resp. odd).

If $[G] \in \pi_{n+1}^{i}(Y)$ and $f_{*}([G])=[f G]=[0]$, then there is $H: 0 \simeq f G$ rel. $i_{n+1}$. Hence, $[(F \kappa, G)] \in \pi_{n+2}^{i}((X, Y))$, where $F$ is an extension of $\{0, H, 0, \ldots, 0\}$ rel. $C i_{n+2}$. So $\delta([(F k, G)])=[G]$.

If $[(F, G)] \in \pi_{n+2}^{i}((X, Y))$ and $[G]=[0] \in \pi_{n+1}^{i}(Y)$, then there is $H: 0 \simeq G$ rel. $i_{n+1}$. Hence, $\left(H^{\prime}, H\right):\left(H^{\prime} C \kappa, 0\right) \simeq(F, G)$ rel. $(C i, i)_{n+1}$, where $H^{\prime}$ is an extension of $\{0, f H, 0, \ldots, 0, F\}$ rel. $\left(C i_{n+1}\right)_{1}$. So $j\left(\left[H^{\prime} C \kappa\right]\right)=[(F, G)]$.

If $[F] \in \pi_{n+2}^{i}(X)$ and $j([F])=[(F, 0)]=[(0,0)]$, then there is $(H, G):(F, 0) \simeq(0,0)$ (resp. $(H, G):(0,0) \simeq(F, 0))$ rel. $(C i, i)_{n+1}$, for $n$ even (resp. odd). Hence, $H$ is an extension of $\{0, f G, 0, \ldots, 0, F, 0\}$ (resp. $\{0, f G, 0, \ldots, 0, F\}$ ) rel. $i_{n+3}$. By Lemma 5.12 it is concluded that $f_{*}([G])=[f G]=[F]$.

Corollary 5.14. For every based object $A$ and pair $(X, Y)$, the following sequence of groups is exact:

$$
\ldots \rightarrow \pi_{3}^{A}(Y) \stackrel{f_{*}}{\rightarrow} \pi_{3}^{A}(X) \stackrel{j}{\rightarrow} \pi_{3}^{A}((X, Y)) \stackrel{\delta}{\rightarrow} \pi_{2}^{A}(Y) \stackrel{f_{*}}{\rightarrow} \pi_{2}^{A}(X)
$$

\section{Algebraic examples}

The homotopy theory developed in the previous sections has a dual version based on the notions of cocone, fibration, nullhomotopy lifting property (NLP) and cosuspension. Cone or cocone structures can be obtained by applying algebraic methods to other homotopy structures. Also, adjointness relations between functors originate cones or cocones.

A cone in a category is a triple $(C, \kappa, \rho)$, in the sense of Definition 3.1, verifying the 
cone axiom. Huber [9] proved that every pair of adjoint functors $(\mathbf{A} \stackrel{U}{\rightarrow} \mathbf{C}, \mathbf{C} \stackrel{V}{\rightarrow} \mathbf{A})$ carries cone structures in $\mathbf{C}$ (resp. cocone structures in $\mathbf{A}$ ) into cone structures in $\mathbf{A}$ (resp. cocone structures in $\mathbf{C}$ ). In this sense, using the trivial cone (resp. trivial cocone) $(1, i d, i d)$ we shall always obtain a cone (resp. cocone) for every pair of adjoint functors. The converse of this fact was proved by Kleisli [13].

Given a cone $(C, \kappa, \rho)$, every right adjoint functor $C^{\prime}$ of $C$ induces a cocone $\left(C^{\prime}, \kappa^{\prime}, \rho^{\prime}\right)$ with adjunction isomorphism identifying $\kappa^{*} \cong \kappa_{*}^{\prime}$ and $\rho^{*} \cong \rho_{*}^{\prime}$. This fact was proved by Huber [9] too.

A morphism $i$ is said to be a cofibration generated by the cone $(C, \kappa, \rho)$ if, for each non-negative integer $n$, the morphism $i_{n}$ verifies the NEP and there exists the pushout object $P\left\{i_{n}, f\right\}$, for any morphism $f$ with the same domain of $i_{n}$.

\subsection{Category with natural spheres (and co-spheres)}

The nth-suspension $S^{n} A$ of a topological space $A$ is obtained by identifying the base of the topological cone $C S^{n-1} A$ to a single point. If $A=S^{0}$ is the 0 -sphere, then $S^{n} A=S^{n}$ is the $n$-sphere. In this way, the homotopy groups referred to an object $A$ can be considered as spherical homotopy groups.

Definition 6.1. A category with natural spheres, or S-category, is a category $\mathbf{C}$ with a cone $(C, \kappa, \rho)$ such that $C$ carries pushouts to pushouts and $\kappa_{A}$ is a cofibration generated by the cone, for every object $A$.

Theorem 6.2. Every $S$-category is a C-category with the cofibrations generated by the cone.

Proof. - Pushout axiom: By hypothesis, if $i: B \longmapsto A$ is a generated cofibration, then there is the pushout object $P\{i, f\}$, for every morphism $f: B \rightarrow X$. Hence, for any morphism $g: X \rightarrow Y$ there exists $P\{\bar{i}, g\}=P\{i, g f\}$. Moreover, $\{C f r, 1\}: P\{C i, C f\} \rightarrow$ $C X$ is a retraction for $C \bar{i}$, where $r$ is a retraction for $C i$. Observing that Lemma 4.12 is also true for diagrams without base morphism, the morphism $\bar{i}$ is a generated cofibration since $\overline{i_{n}}=(\bar{i})_{n}$.

- Cofibration axiom: The development made in the proof of the relative cone axiom in Theorem 5.3 when applied to the cofibration of pairs $\kappa_{(X, Y)}$, proves that $\Sigma^{f_{m}}=$ $P\left\{\kappa_{m}, \Sigma_{m}(C f, f)\right\}$, for every non-negative integer $m$.

Given two generated cofibrations $C \stackrel{j}{\longmapsto} B \stackrel{i}{\longmapsto} A$, the commutative square $1_{A}(i j)=i j$ induces, by Lemma 4.12 , the commutative square $1_{C^{n}(A)}(i j)_{n}=i_{n} \Sigma_{n}\left(1_{A}, j\right)$. In this way, $\Sigma_{n}\left(1_{A}, j\right)=C^{n} j \cup 1: \Sigma^{(i j)_{n-1}}=P\left\{\kappa_{n-1}, \Sigma_{n-1}(C(i j), i j)\right\} \rightarrow \Sigma^{i_{n-1}}=P\left\{\kappa_{n-1}, \Sigma_{n-1}(C i, i)\right\}$. Hence, $(i j)_{n}=i_{n}\left(C^{n} j \cup 1\right)$.

Given a morphism $f=\left\{f_{0}, f_{1}\right\}: \Sigma^{(i j)_{n-1}}=P\left\{\kappa_{n-1}, \Sigma_{n-1}(C(i j), i j)\right\} \rightarrow X$, the following diagram is a pushout:

Hence, the pushout $P\left\{(i j)_{n}, f\right\}$ exists too. 


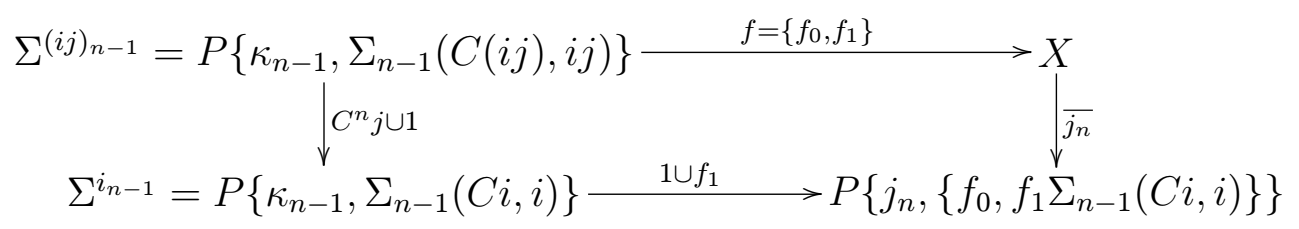

Finally, the morphism $\left\{C\left\{\overline{\Sigma_{n-1}(C i, i)}, \overline{\kappa_{n-1}} \Sigma_{n-1}(C i, i)\right\} r, C \overline{\kappa_{n-1}}\right\}: C \Sigma^{i_{n-1}} \rightarrow C \Sigma^{(i j)_{n-1}}$ is a retraction for $C\left(C^{n} j \cup 1\right)$, where $r C j_{n}=1$. So that $C(i j)_{n}$ is a section.

Remark 6.3. Every $S$-category has homotopy groups relative to generated cofibrations and exact sequences of these groups. In particular, this fact guarantees the existence of the spherical homotopy groups.

The dual concept of a $S$-category is denominated a category with natural co-spheres or $S^{\prime}$-category.

Definition 6.4. A $S S^{\prime}$-category is a category with natural spheres and co-spheres, where $\left(C, C^{\prime}\right)$ is a pair of adjoint functors such that the natural adjunction isomorphism makes $\kappa^{*} \cong \kappa_{*}^{\prime}$ and $\rho^{*} \cong \rho_{*}^{\prime}$.

Remark 6.5. If $\left(C, C^{\prime}\right)$ is a pair of adjoint functors, then $C$ preserves colimits and $C^{\prime}$ preserves limits. Hence, $C$ carries pushouts to pushouts and $C^{\prime}$ carries pullbacks to pullbacks.

Proposition 6.6. In a $S S^{\prime}$-category, the natural adjunction isomorphism $\gamma$ induces an isomorphism $\pi_{n}^{\kappa_{A}}(X) \cong \pi_{n}^{\kappa_{X}^{\prime}}(A)$, for every pair of objects $A, X$.

Proof. The isomorphism $\gamma^{\prime}: \pi_{n}^{\kappa_{A}}(X) \rightarrow \pi_{n}^{\kappa_{X}^{\prime}}(A)$ is defined by $\gamma^{\prime}([F])=\left[\gamma^{n+1}(F)\right]$, where $\gamma^{n+1}$ denote the composite $\gamma \stackrel{(n+1}{\cdots+\cdots} \gamma: \operatorname{Hom}\left(C^{n+1} A, X\right) \rightarrow \operatorname{Hom}\left(A, C^{\prime n+1} X\right)$. In effect, if $F: F_{0} \simeq F_{1}$ rel. $\kappa_{n}$ then $C^{\prime n+1} \kappa^{\prime} F^{\prime}: \gamma^{n+1}\left(F_{0}\right) \simeq \gamma^{n+1}\left(F_{1}\right)$ rel. $\kappa_{n}^{\prime}$, where $F^{\prime}$ is a lifting of the morphism $<\rho^{\prime} \gamma^{n+1}\left(F_{0}\right), C^{\prime n} \rho^{\prime} C^{\prime n} \kappa^{\prime} \rho^{\prime} \gamma^{n+1}\left(F_{0}\right), \ldots . ., C^{\prime n} \rho^{\prime} C^{\prime 2} \kappa^{\prime} \rho^{\prime} \gamma^{n+1}\left(F_{0}\right)$, $C^{\prime n} \rho^{\prime} \gamma^{n+1}\left(F_{0}\right), \gamma^{n+2}(F)>$ relative to the fibration $\left(\kappa_{C^{\prime}}^{\prime}\right)_{n+1}$.

The inverse isomorphism is defined using $\gamma^{-1}$.

The natural adjunction isomorphism $\gamma$ induces, in a natural way, adjunctions between suspensions or relative cones and the respective dual concepts, whose natural isomorphisms will be also denoted by $\gamma$. Moreover, the isomorphism $\gamma$ between $S$ and $S^{\prime}$ preserves the homotopy relation, and so that the classical relation between loops and suspensions can be generalized for $S S^{\prime}$-categories.

Proposition 6.7. Given two objects $A$ and $X$ in a $S S^{\prime}$-category,

$$
\left[S^{n-i} A, S^{\prime m} X\right] \cong\left[S^{n} A, S^{\prime m+i} X\right]
$$

Theorem 6.8. If $\mathbf{C}$ is a S-category with pullbacks and $C^{\prime}$ is a right adjoint functor to 
the cone $C$, then $\mathbf{C}$ is a $S S^{\prime}$-category.

Proof. It suffices to prove that $\kappa_{n}^{\prime}$ verify the NLP.

If $f: A \rightarrow \Sigma_{\kappa_{n}^{\prime}}^{\prime}$ is nullhomotopic, then $F \kappa=f$, for some $F: C A \rightarrow \Sigma_{\kappa_{n}^{\prime}}^{\prime}$. So that $\gamma^{-1}(f)=\gamma^{-1}(F \kappa)=\gamma^{-1}(F) \Sigma_{n}(C \kappa, \kappa): \Sigma^{\kappa_{n-1}} \rightarrow \Sigma^{\left(\kappa_{C}\right)_{n-1}} \rightarrow$ X. Hence, $\gamma^{-1}(f)$ is nullhomotopic, and by the NEP there is a morphism $F: C^{n+2} A \rightarrow X$ such that $F \kappa_{n+1}=\gamma^{-1}(f)$. Therefore, $f=\gamma\left(F \kappa_{n+1}\right)=\left(\Sigma_{n+1}^{\prime}\left(F, C^{\prime} F\right)\right) \gamma\left(\kappa_{n+1}\right) \stackrel{(*)}{=}\left(\Sigma_{n+1}^{\prime}\left(F, C^{\prime} F\right)\right) \kappa_{n+1}^{\prime} \gamma^{n+2}\left(1_{C^{n+2} \mathrm{~A}}\right)=$ $\kappa_{n+1}^{\prime} C^{\prime n+2} F \gamma^{n+2}\left(1_{C^{n+2} \mathrm{~A}}\right)=\kappa_{n+1}^{\prime} \gamma^{n+2}(F)$.

(*) Note that $\gamma^{n+1}\left(C^{n+1-i} \kappa\right)=\gamma^{n+1}\left(1_{C^{n+2} A} C^{n+1-i} \kappa\right)=\gamma^{i}\left(\gamma^{n+1-i}\left(1_{C^{n+2} A}\right) \kappa\right) \stackrel{\left(\kappa^{*} \cong \kappa_{*}^{\prime}\right)}{=}$ $\gamma^{i}\left(\kappa^{\prime} \gamma^{n+2-i}\left(1_{C^{n+2} A}\right)\right)=C^{i} \kappa^{\prime} \gamma^{n+2}\left(1_{C^{n+2} A}\right)$

\subsection{Additive categories}

In [5] we proved that if $\mathbf{C}$ is an additive category, with finite limits and colimits, and, structures of $C$-category and $C^{\prime}$ category on $\mathbf{C}$ such that $\left(C, C^{\prime}\right)$ is a pair of adjoint functors with the natural adjunction isomorphism making $\kappa^{*} \cong \kappa_{*}^{\prime}$, then $\mathbf{C}$ is a proper model category. Moreover, if cofibrations and fibrations are defined by the NEP and the NLP respectively, the model category is also closed. As a consequence of Theorem 6.2 (and its dual) these results can be applied to $S S^{\prime}$-additive categories.

On the other hand, in additive categories the axioms of a $C$-category can be simplified. In this sense, if a category $\mathbf{C}$ has cokernels and a cone $(C, \kappa, \rho)$ carrying pushouts to pushouts, then every morphism verifying the NEP is a cofibration generated by the cone: if $r C i=1$, then $(C \bar{\kappa} \rho+C \bar{i} C r-C \bar{i} C r C \kappa \rho)\left\{C^{2} i, C \kappa\right\}=1$, where $\bar{i}$ and $\bar{\kappa}$ are the induced morphisms in the pushout of $\Sigma^{i}$. Observe that $\mathbf{C}$ has pushouts since it is additive with cokernels, and $\kappa$ is a generated cofibration. Therefore, by Theorem 6.2 , taking the morphisms verifying the NEP as cofibrations, $\mathbf{C}$ is a $C$-category.

Proposition 6.9. Given $C A \stackrel{i}{\longleftarrow} B \stackrel{f_{0}, f_{1}}{\longrightarrow} X$ in a $C$-additive category, then $f_{0} \simeq f_{1}$ rel. $i$ if and only if there is a nullhomotopy $F: f_{1}-f_{0} \simeq 0$ such that $F C i=0$.

Proof. If $H: f_{0} \simeq f_{1}$ rel. $i$, then $H-f_{0} \rho: f_{1}-f_{0} \simeq 0$. Conversely, if $F: f_{1}-f_{0} \simeq 0$, then $F+f_{0} \rho: f_{0} \simeq f_{1}$ rel. $i$.

Remark 6.10. By the previous proposition, $f_{0} \simeq f_{1}$ rel. $i$ if and only if $f_{1}-f_{0} \simeq 0$ rel. $i$. On the other hand, $\operatorname{Hom}(A, X)^{0(i)}$ is a subgroup of $\operatorname{Hom}(A, X)$, for all pairs of objects $A, X$ and morphisms $i$ with codomain $A$. Moreover, if $f_{0} \simeq f_{1}$ rel. $i$ and $g_{0} \simeq g_{1}$ rel. $i$, then $f_{0}-g_{0} \simeq f_{1}-g_{1}$ rel. $i$, since if $F: f_{1}-f_{0} \simeq 0$ rel. $i$ and $G: g_{1}-g_{0} \simeq 0$ rel. $i$, then $F-G:\left(f_{1}-g_{1}\right)-\left(f_{0}-g_{0}\right) \simeq 0$ rel. $i$. Hence, given a cofibration $i: B \longmapsto A$, the set $\left[C^{n} A, X\right]^{0\left(i_{n}\right)}=\operatorname{Hom}\left(C^{n} A, X\right)^{0\left(i_{n}\right)} / \simeq$ rel. $i_{n}$ is an abelian group with the operation induced by the additivity of the category.

Theorem 6.11. In a $C$-additive category, the identity map id $: \pi_{n}^{i}(X) \rightarrow\left[C^{n} A, X\right]^{0\left(i_{n}\right)}$ 
is an isomorphism of groups.

Proof. $[F]+[G]=[\overline{\bar{F}}]+[G]=[\{\bar{F}, 0\} \mu]+[\{0, G\} \mu]=[\{\bar{F}, 0\} \mu+\{0, G\} \mu]=[(\{\bar{F}, 0\}+$ $\{0, G\}) \mu]=[\{\bar{F}, G\} \mu]=[F \cdot G]=[F] \cdot[G]$.

Remark 6.12. Consequently, homotopy groups in a C-additive category are abelian. Thus, we can define the abelian homotopy groups $\pi_{n}^{i}(X)=\left[C^{n} A, X\right]^{0\left(i_{n}\right)}$ and $\pi_{n}^{A}(X)=$ $\pi_{n}^{0_{A}}(X)$, even for $n=0,1$. This definition of homotopy groups in a $C$-additive category renders the condition: "C carries pushouts to pushouts" unnecessary. A $C$-additive category without this condition will be called a $C_{*}$-additive category.

A result similar to Theorem 6.2 is given for $C_{*}$-additive categories.

Theorem 6.13. Given a cone in an additive category $\mathbf{C}$ with cokernels, then $\mathbf{C}$ is a $C_{*}$-additive category with cofibrations defined by the NEP.

Proof. By the properties seen at the beginning of this section it suffices to prove that, given a morphism $i: B \rightarrow A$ verifying the NEP:

The induced morphism $\bar{i}: X \rightarrow P\{i, f\}$ verifies the NEP for every morphism $f: B \rightarrow$ $X$, since $\{g, \kappa\}: P\{i, f\} \rightarrow C X$ extends $\kappa$ rel. $\bar{i}$, where $g$ is an extension of $\kappa f$ rel. $i$.

The morphism $i_{1}: \Sigma^{i} \rightarrow C A$ verifies the NEP, since $(C \bar{\kappa}+\kappa \bar{i} r-C(\bar{i} r \kappa))$ extends $\kappa$ rel. $i_{1}$, where $\bar{i}$ and $\bar{k}$ are the induced morphisms in the pushout of $\Sigma^{i}$ and $r C i=1$.

In this paper, homotopy theory seems to be characterized by contractible objects. This characterisation can be proved in additive categories.

Theorem 6.14. Given an additive category with cokernels. If two cones $(C, \kappa, \rho)$ and $(\widetilde{C}, \widetilde{\kappa}, \widetilde{\rho})$ generate the same contractible objects, then the $C_{*}$ and $\widetilde{C}_{*}$ structures are equivalent.

Proof. Given a morphism $i: B \rightarrow A$, by hypothesis $C B$ and $\widetilde{C} B$ are contractible objects in both theories. Hence, $i$ verifies the NEP with respect to $C$ if and only if $i$ verifies the the NEP with respect to $\widetilde{C}$, and the generated cofibrations are coincident.

Finally, if $F: 0 \simeq f_{1}-f_{0}$ rel. $i$ respect to $\mathbf{C}$, then $F \beta: 0 \simeq f_{1}-f_{0}$ rel. $i$ respect to $\widetilde{C}$, where $\beta$ is an extension of $i_{1}(\alpha \cup 1)$ relative to $\widetilde{i_{1}}$, with $\alpha$ an extension of $\kappa$ relative to $\widetilde{\kappa}$.

The converse is proved in a similar way.

\subsection{Categories with a natural cylinder}

In the Introduction section of this paper, a process to obtain a topological cone starting from a cylinder is described. This fact can be generalized to obtain a $C$-category starting from an $I$-category $\left(\mathbf{C}, \operatorname{cof}, I, \iota_{0}, \iota_{1}, \varrho, \emptyset\right)$ in the sense of Baues [1]. The interchange map $T$ 
used by Baues in the axiom (I5) lets us generalize the natural product of the topological cylinder:

Given an $I$-category, the homotopy extension property (HEP) applied to the homotopy square $\iota_{0} \varrho\left\{\iota_{0}, \iota_{1}\right\}=\left\{\iota_{0} \varrho, 1\right\} \iota_{0}$ induces an extension $F$ verifying $F\left\{I \iota_{0}, I \iota_{1}\right\}=\left\{\iota_{0} \varrho, 1\right\}$ and $F \iota_{0}=\iota_{0} \varrho$. Similarly, the square $\iota_{0} \varrho^{2}\left\{I\left\{\iota_{0}, \iota_{1}\right\}, \iota_{0}, \iota_{1}\right\}=\left\{\iota_{0} \varrho^{2}, F T, \iota_{0} \varrho^{2}, F T\right\} \iota_{0}$ has an extension $H$ verifying $H \iota_{0}=\iota_{0} \varrho^{2}$ and $H I\left\{I\left\{\iota_{0}, \iota_{1}\right\}, \iota_{0}, \iota_{1}\right\}=\left\{\iota_{0} \varrho^{2}, F T, \iota_{0} \varrho^{2}, F T\right\}$.

Hence, $\Psi=H \iota_{1}$ generalizes the fundamental properties of the natural product, that is, $\Psi I \iota_{0}=\iota_{0} \varrho, \Psi I \iota_{1}=1, \Psi \iota_{0}=\iota_{0} \varrho$ and $\Psi \iota_{1}=1$. This fact permits the replacement of the interchange axiom by the following one, without significant changes in the homotopy theory.

(I'5) Product axiom: There exists a natural transformation $\Psi: I^{2} \rightarrow I$ verifying $\Psi I \iota_{1}=\Psi \iota_{1}=1, \Psi I \iota_{0}=\Psi \iota_{0}=\iota_{0} \varrho$ and $\Psi I \Psi=\Psi^{2}$.

An $I$-category where the axiom (I5) is replaced by the axiom $\left(I^{\prime} 5\right)$ is called an $I$ category with a natural product.

Theorem 6.15. Every I-category with a natural product and final object $\varepsilon$ is a $C$ category, with the same cofibrations. Moreover, the homotopies induced by both theories agree.

Proof. The cone functor $C: \mathbf{C} \rightarrow \mathbf{C}$ is defined for objects by $C=P\left\{\iota_{0}, \varepsilon\right\}$ and for morphisms by $I \cup 1_{\varepsilon}$. In this sense, $\overline{\iota_{0}}=C \emptyset: \varepsilon=C \emptyset \rightarrow C$. The natural inclusion is defined by $\kappa=\bar{\varepsilon} \iota_{1}: 1 \rightarrow C$, where $\bar{\varepsilon}$ is the induced morphism in the pushout of the cone. The natural projection is induced by the natural product of the cylinder: $\rho=\left\{\bar{\varepsilon} \Psi, C \emptyset \varepsilon_{I \varepsilon}, C \emptyset\right\}: C^{2} \rightarrow C$, where $\left\{\bar{\varepsilon} \Psi, C \emptyset \varepsilon_{I \varepsilon}\right\}: I C=P\left\{I \iota_{0}, I \varepsilon\right\} \rightarrow C$.

- It is easy to check that $(C, \kappa, \rho)$ is a cone.

- $C$ carries cofibrated pushouts to pushouts by definition, since the cylinder functor $I$ has the required property.

- Observing that objects of an $I$-category are cofibrants, $\kappa_{A}=\left\{C \emptyset_{A}, \kappa_{A}\right\} \overline{\emptyset_{\varepsilon}}: A \longmapsto$ $\varepsilon \sqcup A \longmapsto C A$ is a cofibration since the following diagram is a pushout:

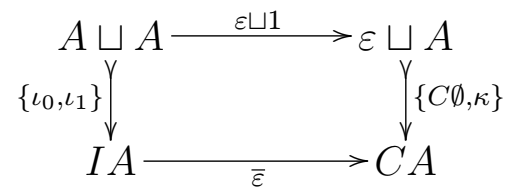

On the other hand, given a cofibration $i: B \longmapsto A$, the homotopy square $\left(C \emptyset_{B} \varepsilon\right) i=$ $C \emptyset_{B} \varepsilon=\bar{\varepsilon} \iota_{0}$ has an extension $F$ verifying $F I i=\bar{\varepsilon}$ and $F \iota_{0}=C \emptyset_{B} \varepsilon$. The morphism $\rho C F C \iota_{1}$ is a retraction for $C i$.

- The morphism $i_{1}$ is a cofibration since the following square is a pushout.

- Recall that $f_{0} \simeq f_{1}$ relative to $i: B \longmapsto A$ respect to a cylinder if and only if there is a morphism $F: I A \rightarrow X$ such that $F\left\{I i, \iota_{0}, \iota_{1}\right\}=\left\{f_{0} i \varrho, f_{0}, f_{1}\right\}$. In particular, if $i: B \longmapsto C A$ is a cofibration:

If $F: f_{0} \simeq f_{1}$ rel. $i$ respect to the cone, then $\left\{f_{0} \rho, F\right\} \widetilde{\kappa} \omega: f_{0} \simeq f_{1}$ rel. $i$ respect to 


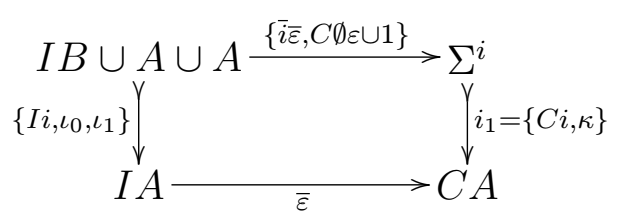

the cylinder, where $\widetilde{\kappa}$ is any extension of $\kappa=\kappa \cup \kappa: P\{i, i\} \rightarrow C(P\{i, i\})=P\{C i, C i\}$ relative to $\overline{\left\{I i, \iota_{0}, \iota_{1}\right\}}$, with $\overline{\left\{I i, \iota_{0}, \iota_{1}\right\}}$ and $\omega$ defined by the following pushout:

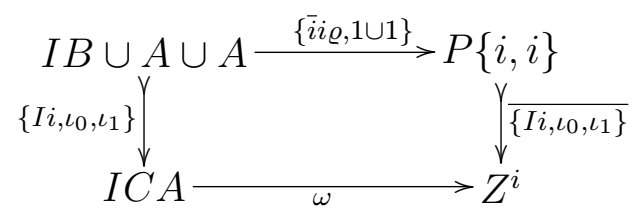

Conversely, given $F: f_{0} \simeq f_{1}$ rel. $i$ respect to the cylinder one can consider the pushout $P\left\{i_{1}, \rho C i \cup 1\right\}$, where $\rho C i \cup 1: \Sigma^{i} \rightarrow P\{i, i\}$. If $H$ is an extension of the homotopy square $\left\{f_{0} \rho, f_{0}, f_{0}\right\} \overline{i_{1}}=\left\{f_{0} \varrho, F\right\} \iota_{0}$, then $H \iota_{1} \overline{\rho C i \cup 1}: f_{0} \simeq f_{1}$ rel. $i$ respect to the cone.

\section{$7 \quad$ Examples}

\subsection{Tensorial homotopies}

Homotopy theories through $S S^{\prime}$-structures are built using the adjunction between the tensorial and Hom functors:

- Every unitary ring $R$ gives rise to a $S S^{\prime}$ structure in the category of abelian groups.

The cone and the cocone of an abelian group $X$ are defined as follows: $C X=X \otimes_{\mathbb{Z}} R$, $\kappa_{X}(x)=x \otimes 1$ and $\rho_{X}(x \otimes r \otimes s)=x \otimes r s ; C^{\prime} X=H_{0} m_{\mathbb{Z}}(R, X) . \quad \kappa_{X}^{\prime}(\alpha)=\alpha(1)$ and $\rho_{X}^{\prime}(\alpha)(r \otimes s)=\alpha(r s)$. The adjunction isomorphism $\gamma$ is defined, for $f: X \otimes R \rightarrow Y$, by $\gamma(f)(x)(r)=f(x \otimes r)$. Observe that, for $g: X \rightarrow Y^{R}$, the inverse is defined by $\gamma^{-1}(g)(x \otimes r)=g(x)(r)$.

An abelian group $X$ is contractible if and only if there is a homomorphism of groups $F: X \otimes R \rightarrow X$ such that $F(x \otimes 1)=x$. This condition is equivalent to the existence of an external operation $G: X \times R \rightarrow X$, denoted by $G(x, r)=r \cdot x$, verifying:
1) $r \cdot\left(x+x^{\prime}\right)=r \cdot x+r \cdot x^{\prime}$
2) $\left(r+r^{\prime}\right) \cdot x=r \cdot x+r^{\prime} \cdot x$
3) $1 \cdot x=x$

These contractible abelian groups are called $R$-quasi-modules.

We point out that not every $R$-quasi-module is a $R$-module. It suffices to consider $R=Q$ the ring of quaternions and $X=O$ the abelian group of octonions (addition of octonions is defined by adding coefficients of the unit octonions $\{1, i, j, k, l, l i, l j, l k\}$, as with the complex numbers and quaternions). The external operation is defined by considering the inclusion $Q \subset O$ and the product of octonions (see the multiplication 
table in http: //en.wikipedia.org/wiki/Octonion, for example). This product verifies that $(i j) l=-i(j l) \neq i(j l)$.

- The category of $R$-quasi modules is also a $S S^{\prime}$-category. A homomorphism of $R$ quasi-modules $f: X \rightarrow Y$ is a homomorphism of groups such that $f(r \cdot x)=r \cdot f(x)$.

If $X$ is a $R$-quasi-module, then so is $X \otimes_{\mathbb{Z}} R$, with the operation generated by $r^{\prime} \cdot(x \otimes$ $r)=x \otimes r^{\prime} r$. Also, $\operatorname{Hom}_{\mathbb{Z}}(R, X)$ is a $R$-quasi-module with the operation $r \cdot \alpha(x)=\alpha(r \cdot x)$. The structure of $S S^{\prime}$-category defined above is also valid in this case.

A $R$-quasi-module $X$ is contractible if and only if $X$ is a $R$-module. Observe that $X \simeq 0$ if and only if there is a homomorphism of $R$-quasi-modules $F: X \otimes R \rightarrow X$ such that $F(x \otimes 1)=x$. Hence, $r^{\prime} \cdot(r \cdot x)=r^{\prime} \cdot F(x \otimes r)=F\left(r^{\prime} \cdot(x \otimes r)\right)=F\left(x \otimes r^{\prime} r\right)=r^{\prime} r \cdot x$.

- Every homomorphism of unitary rings $f: R \rightarrow S$, with $f(1)=1$ and $R$ an abelian ring, induces a $S S^{\prime}$ structure in the category of $R$-modules.

The ring $S$ is a left $R$-module with the operation $r s=f(r) s$. Hence, if $X$ is a $R$ module, then so are $X \otimes_{R} S$ and $\operatorname{Hom}_{R}(S, X)$. A structure of $S S^{\prime}$-category similar to the above is also valid in this case.

A $R$-module $X$ is contractible if and only if $X$ is a $S$-quasi-module. Observe that the first example, viz. abelian groups, is a particular case of this, with $R=\mathbb{Z}$ and $f: \mathbb{Z} \rightarrow S$ defined by $f(n)=n 1$.

\subsection{Chain complexes}

Given an abelian category $\mathbf{A}$, the category of chain complexes over $\mathbf{A}$ is defined as follows. Objects are pairs $(X, \delta)$, where $X=\left\{X_{n}\right\}_{n \in \mathbb{Z}}$ and $\delta=\left\{\delta_{n}\right\}_{n \in \mathbb{Z}}$, with $X_{n}$ objects of $\mathbf{A}$ and $\delta_{n}: X_{n} \rightarrow X_{n-1}$ morphisms verifying $\delta_{n-1} \delta_{n}=0$. If there is no possibility of confusion, we will use the notation $\delta: X_{n} \rightarrow X_{n-1}$. Morphisms from $(X, \delta)$ to $\left(Y, \delta^{\prime}\right)$ are $f=\left\{f_{n}: X_{n} \rightarrow Y_{n}\right\}_{n \in \mathbb{Z}}$ such that $\delta^{\prime} f_{n}=f_{n-1} \delta$.

K. H. Kamps [10] defined a homotopy theory on the category of chain complexes over A by using the cylinder defined by $I(X, \delta)=(I X, I \delta)$, with $(I X)_{n}=X_{n} \oplus X_{n} \oplus X_{n-1}$ and $(I \delta)_{n}=\left(\begin{array}{ccc}\delta & 0 & 1 \\ 0 & \delta & -1 \\ 0 & 0 & -\delta\end{array}\right)$, and given $f:(X, \delta) \rightarrow\left(Y, \delta^{\prime}\right)$ then $(I f)_{n}=\left(\begin{array}{ccc}f_{n} & 0 & 0 \\ 0 & f_{n} & 0 \\ 0 & 0 & f_{n-1}\end{array}\right)$.

The natural transformations are defined by $\iota_{0}=\left(\begin{array}{l}1 \\ 0 \\ 0\end{array}\right), \iota_{1}=\left(\begin{array}{l}0 \\ 1 \\ 0\end{array}\right)$ and $\varrho=\left(\begin{array}{lll}1 & 1 & 0\end{array}\right)$.

This cylinder has a natural product

$$
\Psi_{n}: X_{n} \oplus X_{n} \oplus X_{n-1} \oplus X_{n} \oplus X_{n} \oplus X_{n-1} \oplus X_{n-1} \oplus X_{n-1} \oplus X_{n-2} \rightarrow X_{n} \oplus X_{n} \oplus X_{n-1}
$$

defined by the matrix $\Psi_{n}=\left(\begin{array}{lllllllll}1 & 1 & 0 & 1 & 0 & 0 & 0 & 0 & 0 \\ 0 & 0 & 0 & 0 & 1 & 0 & 0 & 0 & 0 \\ 0 & 0 & 0 & 0 & 0 & 1 & 0 & 1 & 0\end{array}\right)$. Therefore, by Theorem 6.15 there

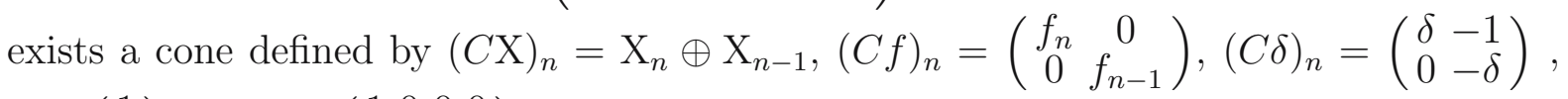
$\kappa=\left(\begin{array}{l}1 \\ 0\end{array}\right)$ and $\rho=\left(\begin{array}{llll}1 & 0 & 0 & 0 \\ 0 & 1 & 1 & 0\end{array}\right)$. 
On the other hand, the homotopy theory defined by Kamps can be obtained via a cocylinder defined by $I^{\prime}(X, \delta)=\left(I^{\prime} X, I^{\prime} \delta\right)$, with $\left(I^{\prime} X\right)_{n}=X_{n} \oplus X_{n} \oplus X_{n+1}$ and given $f:(X, \delta) \rightarrow\left(Y, \delta^{\prime}\right)$ then $\left(I^{\prime} f\right)_{n}=\left(\begin{array}{ccc}f_{n} & 0 & 0 \\ 0 & f_{n} & 0 \\ 0 & 0 & f_{n+1}\end{array}\right)$, where $\left(I^{\prime} \delta\right)_{n}, \iota_{0}^{\prime}, \iota_{1}^{\prime}$ and $\varrho^{\prime}$ are the transpose of $(I \delta)_{n}, \iota_{0}, \iota_{1}$ and $\varrho$, respectively. Moreover, this cocylinder has a natural coproduct $\Psi^{\prime}$, where $\Psi_{n}^{\prime}$ is given by the transpose of $\Psi_{n}$. Hence, there exists a cocone defined by $\left(C^{\prime} \mathrm{X}\right)_{n}=\mathrm{X}_{n} \oplus \mathrm{X}_{n+1},\left(C^{\prime} f\right)_{n}=\left(\begin{array}{cc}f_{n} & 0 \\ 0 & f_{n+1}\end{array}\right)$, and $\left(C^{\prime} \delta\right)_{n}, \kappa^{\prime}$ and $\rho^{\prime}$ are the transpose of $(C \delta)_{n}, \kappa$ and $\rho$, respectively.

We conclude that this homotopy theory can be obtained from a structure of $S S^{\prime}$ category. The natural isomorphism of adjunction $\gamma: \operatorname{Hom}(C-, \sim) \rightarrow \operatorname{Hom}\left(-, C^{\prime} \sim\right)$ is defined by $\gamma\left(f_{n} g_{n-1}\right)=\left(\begin{array}{l}f_{n} \\ g_{n}\end{array}\right)$.

Contractible objects are $(\mathrm{X}, \delta)$ that have associated morphisms $g_{n}: \mathrm{X}_{n} \rightarrow \mathrm{X}_{n+1}$ verifying $\delta g_{n}+g_{n-1} \delta=-1$, for every integer $n$. Generated cofibrations and fibrations are, respectively, the cofibrations and fibrations used by Kamps [10] and defined by the HEP and the HLP, that is, the normal monomorphisms and epimomorphisms, respectively:

Observe that any extension of the morphism $\kappa$ relative to a morphism $i: B \rightarrow A$ verifies $\left(\begin{array}{c}r_{n} \\ r_{n-1}\end{array}\right)\left(i_{n}\right)=\left(\begin{array}{c}r_{n} i_{n} \\ r_{n-1} i_{n}\end{array}\right)=\left(\begin{array}{l}1 \\ 0\end{array}\right)=\kappa_{n}$, for every integer $n$. Hence, $r_{n} i_{n}=1$, and therefore $i_{n}$ is a section, for every integer $n$.

Conversely, if $r_{n}$ is a retraction for $i_{n}$, for every integer $n$, then $\left(\begin{array}{c}r_{n} \\ \delta r_{n}-r_{n-1} \delta\end{array}\right)$ gives an extension of the morphism $\kappa$ relative to the morphism $i$.

\subsection{Pointed topological spaces}

The classical homotopy theory on pointed topological spaces is also generated by a $S S^{\prime}$ structure.

The cone is defined by $C\left(\mathrm{X}, x_{0}\right)=(\mathrm{X} \times I) /\left((\mathrm{X} \times\{0\}) \cup\left(\left\{x_{0}\right\} \times I\right)\right)$, with $\kappa(x)=[x, 1]$ and $\rho([x, t, s])=[x, t s]$. The cocone is defined by $C^{\prime}\left(\mathrm{X}, x_{0}\right)=\operatorname{Hom}\left((I, 0),\left(\mathrm{X}, x_{0}\right)\right)$, with $\kappa^{\prime}(\alpha)=\alpha(1)$ and $\left(\rho^{\prime}(\alpha)\right)(t)(s)=\alpha(t s)$. The natural adjunction isomorphism $\gamma$ is defined by $(\gamma(f))(x)(t)=f([x, t])$. Observe that $\gamma^{-1}\left(f^{\prime}\right)([x, t])=\left(f^{\prime}(x)\right)(t)$.

The product on the cylinder of a topological space mentioned in the Introduction of this paper gives a cone structure on the category of topological spaces. It induces a product on the cylinder of any pointed topological space that originates the above cone. Hence the classical homotopy theory of pointed topological spaces is obtained.

Two cone structures can be associated to topological spaces using HEP cofibrations or closed cofibrations. Contractible topological spaces are the contractible objects of these cone structures. A pointed topological space is contractible if and only if the point is a strong deformation retract of the topological space. 


\subsection{Projective and injective homotopies}

Projective and injective homotopy theories created by Eckmann and Hilton [8] are generated by $C_{*}^{\prime}$ and $C_{*}$ structures, respectively. The cone and cocone structures was defined by Huber [9] using the free, forgetful and Hom functors.

\subsubsection{Projective homotopy theory}

The cocone $C^{\prime} M$ of a $R$-module $M$ is the free $R$-module generated by the pointed set $(M, 0)$. Given a homomorphism of $R$-modules $f: M \rightarrow N$, the cocone $C^{\prime} f: C^{\prime} M \rightarrow C^{\prime} N$ is the unique homorphism of $R$-modules verifying $C^{\prime} f i=j f$, where $i:(M, 0) \rightarrow\left(C^{\prime} M, 0\right)$ and $j:(N, 0) \rightarrow\left(C^{\prime} N, 0\right)$ are the inclusions of pointed sets.

The natural transformations $\kappa^{\prime}$ and $\rho^{\prime}$ are defined as follows: $\kappa_{M}^{\prime}: C^{\prime} M \rightarrow M$ and $\rho_{M}^{\prime}: C^{\prime} M \rightarrow C^{\prime 2} M$ are the unique homomorphisms of $R$-modules such that $\kappa_{M}^{\prime} i=1$ and $\rho_{M}^{\prime} i=j i$, where $i:(M, 0) \rightarrow\left(C^{\prime} M, 0\right)$ and $j:\left(C^{\prime} M, 0\right) \rightarrow\left(C^{\prime 2} M, 0\right)$ are the inclusions of pointed sets.

Contractible objects are projective $R$-modules:

If $M \simeq 0$, then there is $F: M \rightarrow C^{\prime} M$ such that $\kappa^{\prime} F=1$.

Given a homomorphism of $R$-modules $f: M \rightarrow N$ and an epimorphism of $R$-modules $\alpha: L \rightarrow N$. Let $s:(N, 0) \rightarrow(L, 0)$ be a section of $\alpha$ in the category of pointed sets. There is an unique homomorphism of $R$-modules $H: C^{\prime} M \rightarrow L$ such that $H i=s f$. Hence, $\alpha H i=\alpha s f=f=f \kappa^{\prime} i$, and therefore $\alpha H=f \kappa^{\prime}$. So that $\alpha H F=f \kappa^{\prime} F=f$, and $M$ is a projective $R$-module.

The converse is obvious, by observing that $\kappa^{\prime}: C^{\prime} M \rightarrow M$ is an epimorphism of $R$-modules.

\subsubsection{Injective homotopy theory}

The cone of a $R$-module $M$ is defined by $C M=\operatorname{Hom}\left(C^{\prime} \operatorname{Hom}\left(M, Q_{1}\right), Q_{1}\right)$, where $Q_{1}$ is the additive group of the rational numbers module the integers and $C^{\prime}$ is the cocone functor of the above projective homotopy theory. So that

$$
C^{2} M=\operatorname{Hom}\left(C^{\prime} \operatorname{Hom}\left(\operatorname{Hom}\left(C^{\prime} \operatorname{Hom}\left(M, Q_{1}\right), Q_{1}\right), Q_{1}\right), Q_{1}\right)
$$

Observe that the functor $\operatorname{Hom}\left(-, Q_{1}\right)$ carries right (resp. left) modules into left (resp. right) modules. Here we will work in the category of left $R$-modules. A similar development can be achieved for right $R$-modules.

The cone of a homomorphism of $R$-modules $f: M \rightarrow N$ is defined by $C f=\left(C^{\prime} f^{*}\right)^{*}$.

The natural transformations $\kappa$ and $\rho$ are defined as follows:

The homomorphism $\kappa_{M}: M \rightarrow C M$ is such that $\kappa_{M}(m)\left(\bigoplus_{i \in M_{1}} \alpha_{i} r_{i}\right)=\sum_{i \in M_{1}} \alpha_{i} r_{i}(m)=$ $\sum_{i \in M_{1}} \alpha_{i}\left(r_{i} m\right)$ for every finite subset $M_{1} \subseteq \operatorname{Hom}\left(M, Q_{1}\right), m \in M, r_{i} \in R$, and homomorphism of groups $\alpha_{i}: M \rightarrow Q_{1}$. The homomorphism $\rho_{M}: C^{2} M \rightarrow C M$ is such that $\rho_{M}(\alpha)\left(\bigoplus_{i \in M_{1}} \alpha_{i} r_{i}\right)=\alpha\left(\bigoplus_{i \in M_{1}} \widetilde{\alpha}_{i} r_{i}\right)$ for every finite subset $M_{1} \subseteq \operatorname{Hom}\left(M, Q_{1}\right), r_{i} \in R$ 
and homomorphisms of groups $\alpha: C^{\prime} \operatorname{Hom}\left(\operatorname{Hom}\left(C^{\prime} \operatorname{Hom}\left(M, Q_{1}\right), Q_{1}\right), Q_{1}\right) \rightarrow Q_{1}$ and $\alpha_{i}: M \rightarrow Q_{1}$. The homomorphism of groups $\widetilde{\alpha}_{i}: \operatorname{Hom}\left(C^{\prime} \operatorname{Hom}\left(M, Q_{1}\right), Q_{1}\right) \rightarrow Q_{1}$ is defined by $\widetilde{\alpha}_{i}(\beta)=\beta\left(\alpha_{i}\right)$, for every homomorphism of groups $\beta: C^{\prime} \operatorname{Hom}\left(M, Q_{1}\right) \rightarrow Q_{1}$.

Contractible objects are injective $R$-modules:

To prove that every contractible $R$-module is injective, first we will see that $C M$ is an injective module, for every $R$-module $M$.

Since the tensor product is distributive with respect to direct sums, the tensor product of a module with its ring is isomorphic to the module, and the modules $C^{\prime} M$ and $\bigoplus_{M} R$ are isomorphic, for every $R$-module $M$, hence:

$$
-\otimes_{R} C^{\prime} M \cong-\otimes_{R}\left(\bigoplus_{M} R\right) \cong-\bigoplus_{M}\left(-\otimes_{R} R\right) \cong \bigoplus_{M}-
$$

On the other hand, $-\otimes_{R} N$ is a left adjoint functor of $\operatorname{Hom}_{\mathbb{Z}}(N,-)$, for every $R$-module $N$. In particular $\bigoplus_{M}-$ is a left adjoint functor of $\operatorname{Hom}_{\mathbb{Z}}\left(C^{\prime} M,-\right)$.

Since $Q_{1}$ is an injective abelian group, using the adjunction isomorphism, the $R$ module $\operatorname{Hom}_{\mathbb{Z}}\left(C^{\prime} M, Q_{1}\right)$ is injective, for every $R$-module $M$. In particular, so is $C M=$ $\operatorname{Hom}\left(C^{\prime} \operatorname{Hom}\left(M, Q_{1}\right), Q_{1}\right)$.

If the $R$-module $M$ is contractible, then there is a homomorphism of $R$-modules $F: C M \rightarrow M$ such that $F \kappa_{M}=1_{M}$. Given a homomorphism of $R$-modules $f: N \rightarrow M$ and a monomorphism of $R$-modules $\alpha: N \rightarrow L$, then, since $C M$ is an injective module, there is a homomorphism of $R$-modules $\tilde{f}: L \rightarrow C M$ such that $\tilde{f} \alpha=\kappa_{M} f$. Hence, $F \widetilde{f} \alpha=F \kappa_{M} f=f$.

Conversely, since $M$ is injective and $\kappa_{M}: M \rightarrow C M$ is an injective homomorphism of $R$-modules, there is $F: C M \rightarrow M$ such that $F \kappa_{M}=1$.

Observe that the homomorphism of $R$-modules $\kappa_{M}: M \rightarrow C M$ is injective. Since $Q_{1}$ is an injective abelian group, for every abelian group $M$ and $0 \neq m \in M$ there is a homomorphism of groups $\alpha_{m}: M \rightarrow Q_{1}$ such that $\alpha_{m}(m) \neq 0$. If $\kappa(m)=0$, then $m=0$, since if $m \neq 0$, then there is $\alpha_{m}: M \rightarrow Q_{1}$ such that $\kappa(m)\left(\alpha_{m}\right)=\alpha_{m}(m) \neq 0$.

\subsection{Exterior spaces}

In general, the category $\mathbf{P}$ of topological spaces and proper maps does not have limits and colimits. In particular, pushouts do not always exist. However, $\mathbf{P}$ is a full subcategory of the category $\mathbf{E}$ of exterior spaces, and the pushout of every pair of morphisms exists in $\mathbf{E}$. This fact allows the construction of a structure of natural cone through a cylinder with natural product. Since both homotopy theories agree (via the cylinder and via the cone) on the full subcategory $\mathbf{P}$, we obtain the proper homotopy of topological spaces (see $[6])$.

The category of exterior spaces is defined as follows. An externology for a topological space $(X, \tau)$ is a subset $\varepsilon \subset \tau$ verifying the properties E1 and E2:

E1. If $E, E^{\prime} \in \varepsilon$, then $E \cap E^{\prime} \in \varepsilon$. 
E2. If $A \in \tau, E \in \varepsilon$ and $E \subset A$, then $A \in \varepsilon$.

An exterior space $(X, \tau, \varepsilon)$ is a topological space $(X, \tau)$ with an externology $\varepsilon$. An exterior morphism $f:(X, \tau, \varepsilon) \rightarrow\left(X^{\prime}, \tau^{\prime}, \varepsilon^{\prime}\right)$ is a continuous map $f:(X, \tau) \rightarrow\left(X^{\prime}, \tau^{\prime}\right)$ such that $f^{-1}\left(E^{\prime}\right) \in \varepsilon$, for every $E^{\prime} \in \varepsilon^{\prime}$.

The category of exterior spaces has pushout objects: Given two exterior morphisms $f:(X, \tau, \varepsilon) \rightarrow\left(X^{\prime}, \tau^{\prime}, \varepsilon^{\prime}\right)$ and $g:(X, \tau, \varepsilon) \rightarrow\left(X^{\prime \prime}, \tau^{\prime \prime}, \varepsilon^{\prime \prime}\right)$, the pushout object $P\{f, g\}$ is the topological space $P\{f, g\}$ with the externology defined by

$$
\left\{E \subset P\{f, g\} / \bar{f}^{-1}(E) \in \varepsilon^{\prime \prime} \text { and } \bar{g}^{-1}(E) \in \varepsilon^{\prime}\right\}
$$

On the other hand, given two exterior spaces $(X, \tau, \varepsilon)$ and $\left(X^{\prime}, \tau^{\prime}, \varepsilon^{\prime}\right)$, the exterior product space is the topological product space $\left(X \times X^{\prime}, \tau^{\prime \prime}\right)$ with the externology $\varepsilon^{\prime \prime}=$ $\left\{E^{\prime \prime} \in \tau^{\prime \prime} /\right.$ there are $E \in \varepsilon$ and $E^{\prime} \in \varepsilon^{\prime}$ such that $\left.E \times E^{\prime} \subset E^{\prime \prime}\right\}$.

The cylinder of an exterior space $(X, \tau, \varepsilon)$ is the exterior product space $X \times I$, where the externology of the unit interval is $\{I\}$. In this way, the natural transformations $\iota_{0}$, $\iota_{1}, \varrho, \Psi$ given in the category of topological spaces are exterior morphisms. Hence, a cylinder structure with a natural product is obtained in the category of exterior spaces.

A cone structure is obtained similarly as in the category of topological spaces. Observe that the cone of an exterior space $(X, \tau, \varepsilon)$ is the topological cone $\left(C X, \tau^{\prime}\right)$ with the externology $\left\{E^{\prime} \in \tau^{\prime} /\right.$ there is $E \in \varepsilon$ such that $\left.C E \subset E^{\prime}\right\}$.

\section{References}

[1] H.J. Baues: Algebraic homotopy, Cambridge Studies in Advanced Mathematics 15, Cambridge University Press, Cambridge-New York, 1989.

[2] H.J. Baues and A. Quintero: "On the locally finite chain algebra of a proper homotopy type", Bull. Belg. Math. Soc. Simon Stevin, Vol. 3(2), (1996), pp. 161-175.

[3] F.J. Díaz and S. Rodríguez-Machín: "Homotopy theory induced by cones", Extracta Math., Vol. 16(2), (2001), pp. 287-292.

[4] F.J. Díaz, J. Remedios and S. Rodríguez-Machín: "Generalized homotopy in Ccategories", Extracta Math., Vol. 16(3), (2001), pp 393-403.

[5] F.J. Díaz, J. García-Calcines and S. Rodríguez-Machín: Homotopía algebraica: descripción e interrelación de las principales teorías, Monografías de la Academia de Ciencias Exactas, Físicas, Químicas y Naturales de Zaragoza 5, 1994.

[6] J. García-Calcines, M. García-Pinillos and L.J. Hernández-Paricio: "A closed simplicial model category for proper homotopy and shape theories" B. Aust. Math. Soc., Vol. 57, (1998), pp. 221-242.

[7] L.J. Hernández: Un ejemplo de Teoría de homotopía en los grupos abelianos, Departamento de Geometría y Topología, Universidad de Zaragoza, 1980.

[8] P.J. Hilton: Homotopy theory and duality, Gordon and Breach Science Publishers, New York-London-Paris, 1965.

[9] P.J. Huber: "Homotopy theory in general categories", Math. Ann., Vol. 144, (1961), pp. 361-385. 
[10] K.H. Kamps: "Note on normal sequences of chain complexes", Colloq. Math., Vol 39(2), (1978), pp. 225-227.

[11] K.H. Kamps and T. Porter: Abstract Homotopy and Simple Homotopy, World Scientific Publishing Co., Inc., River Edge, NJ, 1997.

[12] H. Kleisli: "Homotopy theory in Abelian Categories", Canad. J. Math., Vol. 14, (1962), pp. 139-169.

[13] H. Kleisli: "Every Standard construction is induced by a pair of Adjoint Functors", Proc. Am. Math. Soc., Vol 16(3), (1965), pp. 544-546.

[14] E.G. Minian: "Generalized cofibration categories and global actions", Special issues dedicated to Daniel Quillen on the occasion of his sixtieth birthday, Part I. K-Theory, Vol 20(1), (2000), pp. 37-95.

[15] T. Porter: "Abstract Homotopy Theory: The Interaction of Category Theory and Homotopy", Cubo Mat. Educ., Vol 5(1), (2003), pp. 115-165.

[16] E. Padrón and S. Rodríguez-Machín: "Model additive categories", Rend. Circ. Mat. Palermo, Suppl., Vol. 24, (1990), pp. 465-474.

[17] D.G. Quillen: Homotopical Algebra, Lecture Notes in Math, Vol. 43, Springer-Verlag, Berlin-New York, 1967.

[18] J.A. Seebach Jr.: "Injectives and homotopy", Illinois J. Math., Vol. 16, (1972), pp. 446-453. 